\title{
Nonlinear Seismic Response Characteristics of CAP1400 Nuclear Island Structure on Soft Rock Sites
}

\author{
Furong $\mathrm{Li} \mathbb{D}^{1,2}$ and Guoxing Chen $\mathbb{D}^{1}$ \\ ${ }^{1}$ Institute of Geotechnical Engineering, Nanjing Tech University, Nanjing 210009, China \\ ${ }^{2}$ College of Civil Engineering, Yancheng Institute of Technology, Yancheng 224051, China \\ Correspondence should be addressed to Guoxing Chen; gxchen@njtech.edu.cn
}

Received 18 March 2020; Revised 26 June 2020; Accepted 10 September 2020; Published 22 September 2020

Academic Editor: Arkady Serikov

Copyright (c) 2020 Furong Li and Guoxing Chen. This is an open access article distributed under the Creative Commons Attribution License, which permits unrestricted use, distribution, and reproduction in any medium, provided the original work is properly cited.

\begin{abstract}
CAP1400 nuclear island structure is an advanced and novel nuclear power plant structure. In order to explore the seismic response characteristics of CAP1400 nuclear island structure on soft rock sites, a three-dimensional refined nonlinear seismic response analysis model was established for a soft rock foundation-nuclear island structure system using ABAQUS software. The influences of the input ground motion intensity and the frequency spectrum characteristics on the acceleration, relative displacement, and floor response spectrum, as well as the critical shear wave velocity of nonbedrock sites for CAP1400 nuclear island structure, were proposed. The results suggested that the increasing amplitude of the peak acceleration and relative displacement of nuclear island structure decreased as the soft rock site entered a nonlinear state, and the high-frequency components of the input ground motion became more abundant. Specifically, the earthquake response was the largest at the cooling water tank on the top of the shield building, which was the focus of the seismic research on nuclear island structure. Due to the influence of the ground motion frequency spectrum characteristics and the nonbedrock site effect, the peak acceleration, peak relative displacement, and acceleration response spectrum of the nuclear island structure showed different changing trends for the near-field and far-field ground motions. Based on the influence of the site shear wave velocity on the seismic response of nuclear island structure, it was recommended that the critical shear wave velocity of nonbedrock sites for CAP1400 nuclear island structure should be $1250 \mathrm{~m} / \mathrm{s}$, and the nuclear island structure-foundation dynamic interaction could be ignored at this time. The research conclusions could provide some technical support and theoretical basis for the construction and seismic performance research of CAP1400 and other nuclear power plants.
\end{abstract}

\section{Introduction}

According to the statistics of the International Atomic Energy Agency, nuclear power generation accounts for about $10 \%$ of global power generation and nearly $1 / 3$ of lowcarbon power generation. Additionally, nuclear power generation is an important part of the global energy strategy. Nuclear power safety is a top priority in the development of nuclear power, and the impact of earthquakes is an important consideration for nuclear power safety. CAP1400 is the third generation of advanced nuclear power technology in China's nuclear power plant construction, and it is the mainstream direction for the future large-scale development of nuclear power in the world. Compared with other nuclear power structures, CAP1400 nuclear island structure has the characteristics of larger size, higher power, and a more complex structure, which puts forward new requirements and new challenges for research on the seismic performance of CAP1400 nuclear island structure. Currently, there is only one CAP1400 nuclear power plant under construction in the world, built on the hard bedrock site $\left(V_{s}>2438 \mathrm{~m} / \mathrm{s}\right)$ of Shidao Bay, Shandong Province, China $[1,2]$. With the rapid development of global nuclear power plant construction, high-quality bedrock sites are becoming more and more scarce, and nuclear power plants will be gradually built on nonbedrock sites such as soft rock and soil [3-5]. Therefore, the earthquake safety of CAP1400 nuclear island structure on nonbedrock sites urgently needs in-depth systematic 
research to provide the necessary theoretical basis for the construction and seismic performance research of nuclear power projects.

In recent years, scholars have paid close attention to the seismic responses and seismic performances of nuclear power plants, and scholars have carried out extensive preliminary studies on the soil-structure interaction (SSI) of a nuclear island structure. For example, Tuñón-Sanjur et al. analyzed the seismic response characteristics of AP1000 nuclear island structure on general hard bedrock sites using ANASYS finite element software, and they designed five types of nonbedrock sites that needed to consider SSI [1]. Ashiquzzaman and Hong et al. established relatively simple models for the containment vessel of a nuclear power plant (e.g., the particle-beam model) and analyzed the impact of SSI on the seismic response characteristics of the containment structure [6-8]. While evaluating the seismic safety performance of Japanese nuclear power structures, Nakamura et al. conducted a seismic response analysis of nuclear island structures by considering SSI [9]. Kim et al. adopted a new boundary condition to analyze the seismic response characteristics of nuclear reactor structures under SSI in which the structure adopted a concentrated mass model [10]. Wang and Yang established an AP1000 nuclear island finite element model and analyzed the seismic response of a nuclear island structure under different foundation conditions by considering the foundation embedment and SSI [11]. Li et al. established a finite element model by considering the foundation-nuclear island structure interaction, and they analyzed the seismic response of the contact area between the shield building and the foundation soil for different types of sites and different ground motion inputs [12]. It can be seen that, for the current research, the seismic response characteristics of CAP1400 nuclear island structure on nonbedrock sites are rarely involved, and the corresponding critical shear wave velocity of nonbedrock sites needs to be studied and given.

In this research, the actual soft rock site of a nuclear power plant was selected based on ABAQUS finite element software, with consideration of the nonlinear characteristics of soft rock and a viscoelastic artificial boundary. A threedimensional refined model of CAP1400 nuclear island structure-foundation dynamic interaction was established, and then the seismic response characteristics of CAP1400 nuclear island structure system were analyzed. According to the influence of the shear wave velocity on the seismic response characteristics of the nuclear island structure, the critical shear wave velocity of nonbedrock sites suitable for CAP1400 nuclear island structure was proposed. This research provides a theoretical basis for nuclear power plant construction and seismic performance research.

\section{Finite Element Model of CAP1400 Nuclear Island Structure}

2.1. Overview of Nuclear Island Structure. CAP1400 nuclear island structure is mainly composed of the shield building $(\mathrm{SB})$, auxiliary building $(\mathrm{AB})$, steel containment vessel (SCV), and containment internal structure (CIS) (Figure 1).

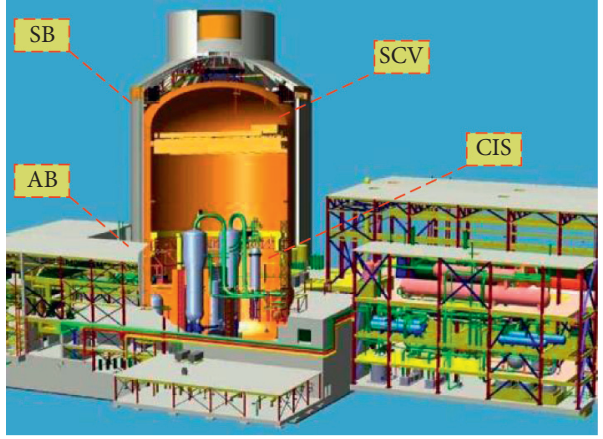

Figure 1: Diagram of the nuclear island structure.

The entire structure is located on a $1.8 \mathrm{~m}$ thick raft foundation, and it is a Class I seismic structure. The nuclear island structure has a height of about $87.75 \mathrm{~m}$ (including the basement and a buried depth of $11.7 \mathrm{~m}$ ), a length of $90.80 \mathrm{~m}$, and a width of $57.70 \mathrm{~m}$. The outer diameter of the $\mathrm{SB}$ is about $47.97 \mathrm{~m}$, and the wall thickness is $1.10 \mathrm{~m}$. The $A B$ is a reinforced concrete shear wall structure with elevations of $18.05 \mathrm{~m}, 20.35 \mathrm{~m}$, and $35.30 \mathrm{~m}$; it contains a two-level basement, and the bottom elevation is $-11.70 \mathrm{~m}$. SCV has a height of about $65.70 \mathrm{~m}$ and a diameter of $39.70 \mathrm{~m}$. A crane beam is coupled to the SCV, with an elevation of $39.0 \mathrm{~m}$.

2.2. Establishment of Finite Element Model. A refined model of CAP1400 nuclear island structure was established using ABAQUS finite element software. The $\mathrm{SB}, \mathrm{AB}$, and SCV were simulated with the shell element (S4R). The concrete base floor and the concrete foundation inside the SCV were simulated with the solid element (C3D8R). The equipment installation floor inside the SCV and the nuclear island raft foundation were simulated with the shell elements (S4R). The internal reactor cooling loop (RCL), pressurizers, and so forth were simulated by the three-dimensional beam element (B31) and connected to the internal structure through the rod element (T3D2). The Tie constraint was adopted between the SB and CIS and the AB, and the Embedded constraint was adopted between the SCV and CIS. Figure 2 illustrates the refined finite element model of the nuclear island structure, which adopted CAP1400 structural design parameters, that is, the weights of the concrete structure and the SCV steel plate were $2550 \mathrm{~kg} / \mathrm{m}^{3}$ and $7800 \mathrm{~kg} / \mathrm{m}^{3}$, the elastic moduli were $34.5 \mathrm{GPa}$ and $206 \mathrm{GPa}$, and Poisson's ratios were 0.2 and 0.3 , respectively.

2.3. Modal Analysis of Nuclear Island Structure. In order to better analyze the dynamic characteristics of the nuclear island structure, the Lanczos method was adopted to perform a modal analysis on CAP1400 structure [13, 14]. According to $[15,16]$, the first ten modes were chosen for extraction in this research, and it was found that the natural vibration period of the first two modals of the nuclear island structure was about $0.23 \mathrm{~s}$ (Table 1). The modes were mainly expressed as the beam translation in the $Y$ - and $X$-directions (Figure 3). It could be seen that the horizontal ground motion had a greater impact on the nuclear island structure, 


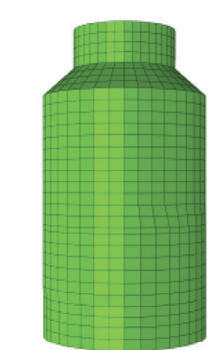

SB

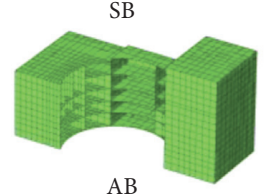

$\mathrm{AB}$

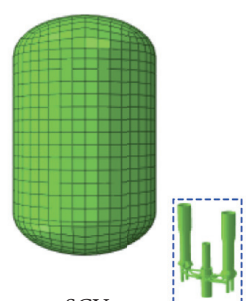

SCV

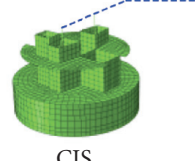

CIS

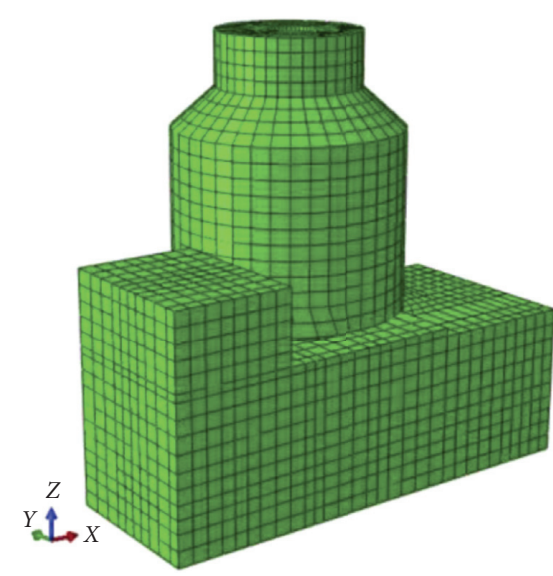

(b)

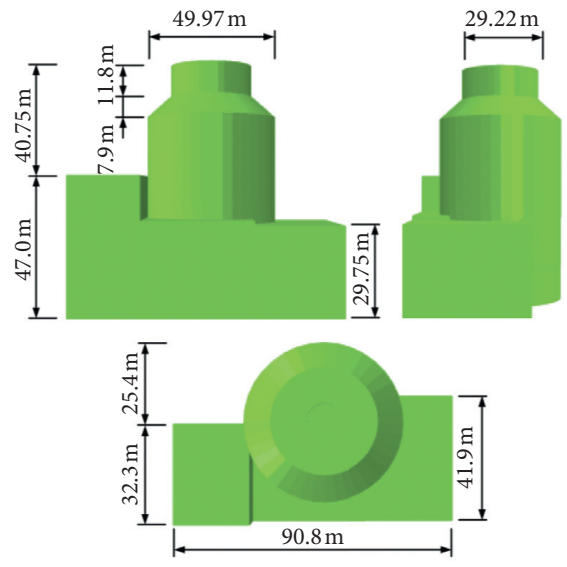

(c)

FIgUre 2: Finite element model of the nuclear island structure. (a) Model composition. (b) Whole model. (c) Three views.

TABLE 1: First ten modes of CAP1400 nuclear island structure.

\begin{tabular}{|c|c|c|c|c|c|c|c|c|c|c|}
\hline Mode & $1 \mathrm{st}$ & 2nd & $3 \mathrm{rd}$ & 4 th & 5 th & 6th & 7th & 8th & 9th & 10th \\
\hline Period (s) & 0.2345 & 0.2234 & 0.1546 & 0.1426 & 0.1381 & 0.1329 & 0.1185 & 0.1157 & 0.1123 & 0.1107 \\
\hline
\end{tabular}

$\mathrm{U}$, Magnitude

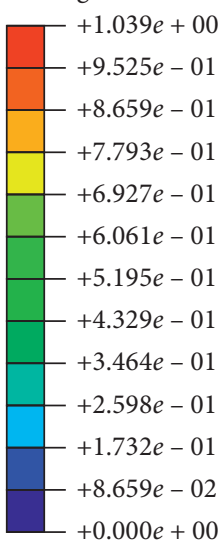

U, Magnitude

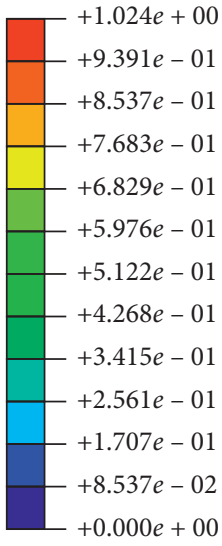

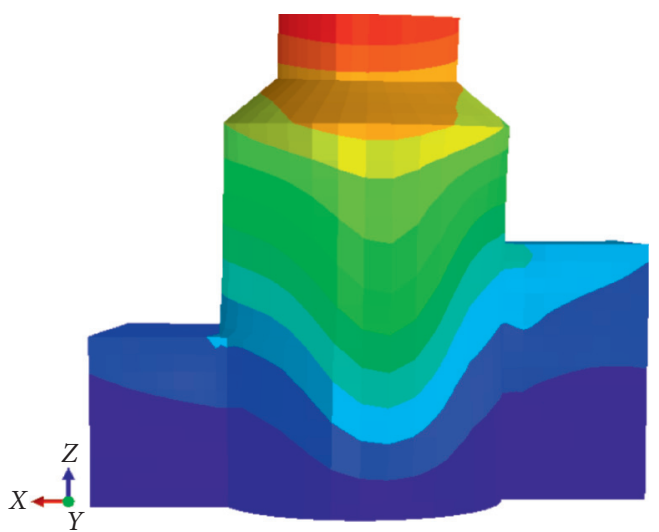
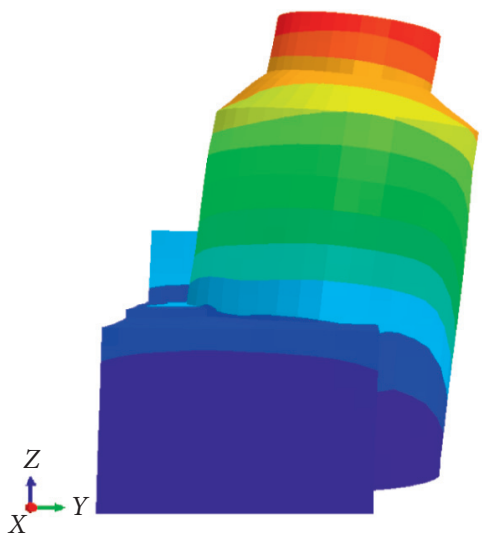

(a)
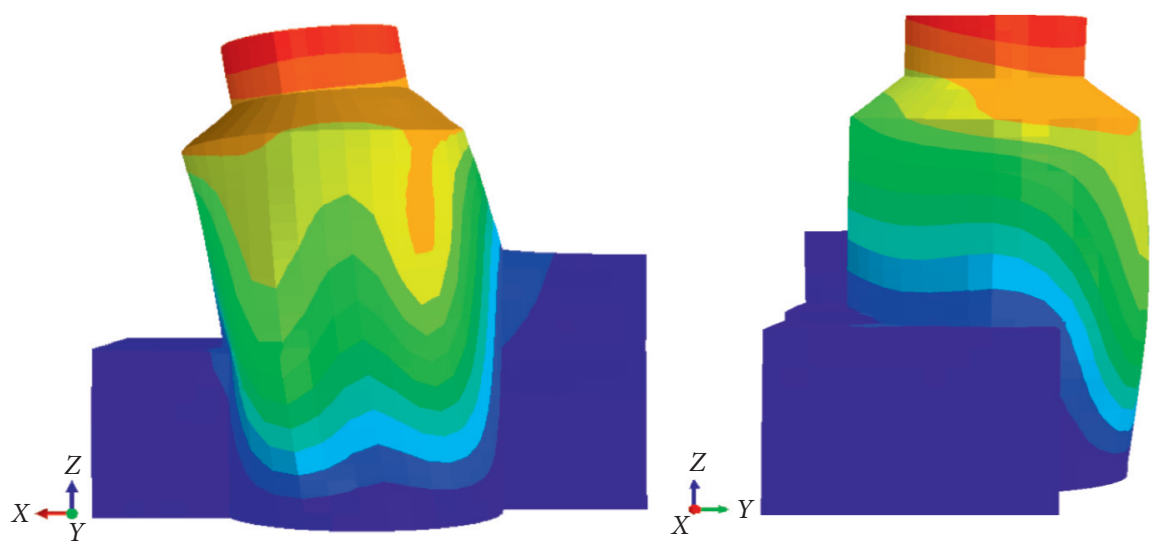

(b)

FIgURE 3: The first two modes of CAP1400 nuclear island structure. (a) The 1st modal. (b) The 2nd modal. 
and the maximum deformation occurred at the cooling water tank on the top of the SB. The first and second order modes of vibration revealed the stiffness of CAP1400 nuclear island structure model, that is, the stiffness in the $Y$-direction was slightly smaller than the stiffness in the $X$-direction.

The SB was constrained by the surrounding C-shaped $A B$, which enhanced the stiffness on one side of the nuclear island structure. Its first-order mode was along the direction of weaker stiffness, indicating that the $A B$ changed the dynamic characteristics of the nuclear island structure, and the influence of the $\mathrm{AB}$ on the dynamic characteristics of the entire nuclear island structure could not be ignored. The entire nuclear island had to be considered to accurately reflect its seismic response characteristics, which was consistent with the research conclusions in [17].

In order to ensure the correctness of the calculation model, the modal analysis results of CAP1400 nuclear island structure were compared with the results of the relevant existing literature $[11,18-22]$. As can be seen from Table 2, the natural vibration frequencies of the first two orders were similar to the results reported in the existing literature. Specifically, the average natural vibration frequencies of the first two orders given by the existing literature were $4.17 \mathrm{~Hz}$ and $4.30 \mathrm{~Hz}$, respectively. Compared with the nuclear island structure model in this research, the error rates were only $2.17 \%$ and $4.02 \%$. Therefore, the finite element model of CAP1400 nuclear island structure established in this research was more reasonable.

\section{Dynamic Calculation Model of the Soft Rock Foundation-Nuclear Island Structure System}

3.1. Soft Rock Sites. AP1000 standard design provides five types of nonbedrock sites with shear wave velocities of less than $1100 \mathrm{~m} / \mathrm{s}$, that is, firm rock (FR), soft rock (SR), upper bound soft-to-medium soil (SMS-UB), soft-to-medium soil (SMS), and soft soil (SS), in addition to hard bedrock [1]. Figure 4 shows the shear wave velocity for these five types of nonbedrock sites as a function of depth. Specifically, the shear wave velocity of SR is about $740-960 \mathrm{~m} / \mathrm{s}$. According to "the Safety Guidelines for Site Evaluation and Foundation Geotechnical Engineering Issues of Nuclear Power Plants," an SR site belongs to the Class II site foundation category, and its shear wave velocity was about $300-1100 \mathrm{~m} / \mathrm{s}$. During nuclear power plant site selection, the shear wave velocity of the currently selected SR sites mostly ranges from $350 \mathrm{~m} / \mathrm{s}$ to $950 \mathrm{~m} / \mathrm{s}$, with the sites being mainly mudstone or muddy cemented sandstone $[23,24]$.

During site selection of a nuclear power plant, SR is a good option for a nonbedrock site. However, as one of the five types of AP1000 nonbedrock sites, SR has some special engineering properties, which are that SR is equivalent to general strongly weathered rocks, and it has nonlinear and strain softening characteristics [25]. At present, the seismic analysis of nuclear island structures on SR sites is a frontier research topic.

In this research, the upper stratum of the site selected for the nuclear power plant was the Quaternary Neolithic SeaLand Alternate Faces sedimentary layer, the soil was mainly silty clay, with silt sand and silt soil in part, and the exposed thickness was in the range of 8.0-25.5 $\mathrm{m}$. The lower part was composed of strongly weathered mudstone, moderately weathered mudstone, sandstone, and so forth, which were continuously distributed and horizontally layered. This site belonged to the category of a typical SR site. The basic mechanical parameters of the site are shown in Table 3. According to the "Code for Seismic Design of Buildings" (GB50011-2010), the equivalent shear wave velocity of the site was $515 \mathrm{~m} / \mathrm{s}$, which meant that the site was categorized as a Class $I_{1}$ site.

3.2. Nonlinear Constitutive Model. While analyzing the SR foundation-nuclear island structure dynamic interaction, the modified Davidenkov constitutive model that was secondarily developed by Zhao et al. was used to describe the dynamic nonlinear characteristics of the SR foundation [26]. This constitutive model has been used for the seismic response analysis of soil-underground structures, and it has a certain degree of reliability [27-29]. The stress-strain relationship of the constitutive model is shown in Figure 5(a).

The relationship between the dynamic shear modulus ratio $G / G_{\max }$, the damping ratio $\lambda$, and the shear strain $\gamma$ for the SR site foundation was obtained with the dynamic triaxial test [30], as shown in Figure 5(b). The Davidenkov backbone curve parameters $A, B$, and $\gamma_{0}$ are shown in Table 4 [31], as obtained by fitting equation:

$$
\frac{G}{G_{\max }}=1-\left(\frac{\left(\gamma / \gamma_{0}\right)^{2 B}}{1+\left(\gamma / \gamma_{0}\right)^{2 B}}\right)^{A} .
$$

3.3. Mesh Generation and Boundary Condition. The maximum vertical dimension $h_{\max }$ of the mesh of the SR foundation model was taken as $1 / 8$ to $1 / 10$ of the wavelength corresponding to the cutoff frequency [32], which could be calculated based on the following equation:

$$
\frac{V_{s}}{10 f_{\max }} \leq h_{\max } \leq \frac{V_{s}}{8 f_{\max }},
$$

where $V_{s}$ is the shear wave velocity of the soil layer and $f_{\max }$ is the cutoff frequency, which was taken as $25 \mathrm{~Hz}$. According to this, the vertical dimension of the mesh was in the range of $1.3-4.0 \mathrm{~m}$, the horizontal dimension was in the range of $1.5-3.0 \mathrm{~m}$, and the mesh of the area near the nuclear island structure was refined.

In order to eliminate the boundary effect caused by the reflection and refraction of seismic waves at the SR site foundation model boundary, the lateral size of the SR foundation model was about five times the foundation size of the nuclear island structure, and the viscoelastic artificial boundary was adopted [33]. The buried depth of the nuclear island structure model was $11.7 \mathrm{~m}$. The structure was connected to the SR site by the Tie constraint. Figure 6 illustrates the overall established refined model of the SR foundationnuclear island structure system. 
TABLE 2: Comparison of the first two order modes of the nuclear island structure model.

\begin{tabular}{|c|c|c|c|c|c|c|c|c|}
\hline \multirow{2}{*}{ Type } & \multicolumn{3}{|c|}{ Nuclear island structure (m) } & \multirow{2}{*}{ Calculation platform } & \multicolumn{4}{|c|}{ Natural vibration frequency $(\mathrm{Hz})$} \\
\hline & Height & Diameter & Thickness & & $1 \mathrm{st}$ & Error & 2nd & Error \\
\hline CNP1000 (Duan et al. [18]) & 48.00 & 40.00 & 1.10 & MSC. MARC & 4.34 & $1.88 \%$ & 4.38 & $2.23 \%$ \\
\hline CNP1000 (Zhang et al. [19]) & 48.00 & 40.00 & 1.10 & SAP2000 & 4.48 & $5.16 \%$ & 4.52 & $0.89 \%$ \\
\hline CPR1000 (Yi et al. [20]) & 66.68 & 37.00 & 0.90 & ANASYS & 4.22 & $0.94 \%$ & 4.28 & $4.46 \%$ \\
\hline AP1000 (Wang and Yang [11]) & 83.36 & 44.20 & 1.00 & ABAQUS & 3.92 & $7.98 \%$ & 4.25 & $5.13 \%$ \\
\hline AP1000 (Wang et al. [21]) & 83.37 & 44.2 & 0.912 & ANASYS & 4.04 & $5.16 \%$ & 4.04 & $9.82 \%$ \\
\hline CAP1400 (Li et al. [22]) & 87.75 & 49.97 & 1.10 & ABAQUS & 3.99 & $6.34 \%$ & 4.35 & $2.90 \%$ \\
\hline CAP1400 (this research) & 87.75 & 49.97 & 1.10 & ABAQUS & 4.26 & - & 4.48 & - \\
\hline
\end{tabular}

Note. Error $=($ calculation result of existing literature - calculation result in this research $) /$ calculation result in this research $\times 100 \%$.

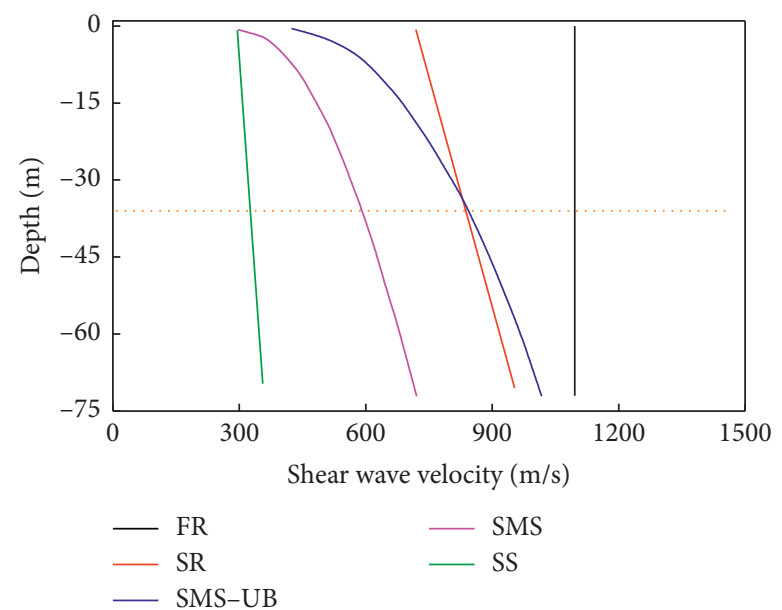

FIGURE 4: Shear wave velocity curve of nonbedrock sites in AP1000 standard design.

TABLE 3: Basic mechanical parameters of the SR site.

\begin{tabular}{lcccc}
\hline Soils & Thick $(\mathrm{m})$ & Density $\left(\mathrm{kg} / \mathrm{m}^{3}\right)$ & Shear wave velocity $(\mathrm{m} / \mathrm{s})$ & Poisson's ratio $\mu$ \\
\hline Silty clay & 10.2 & 1960 & 316 & 0.47 \\
Strongly weathered mudstone & 9.4 & 2110 & 461 & 0.39 \\
Moderately weathered mudstone & 13.9 & 2250 & 559 & 0.35 \\
Sandstone & 16.5 & 2300 & 807 & 0.32 \\
\hline
\end{tabular}

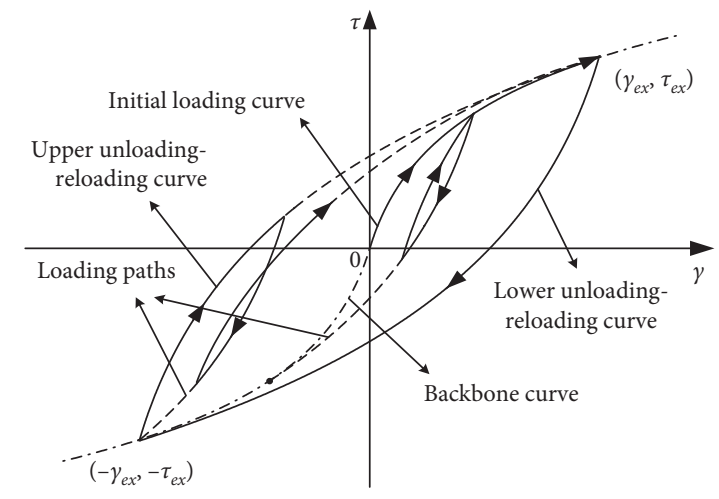

(a)

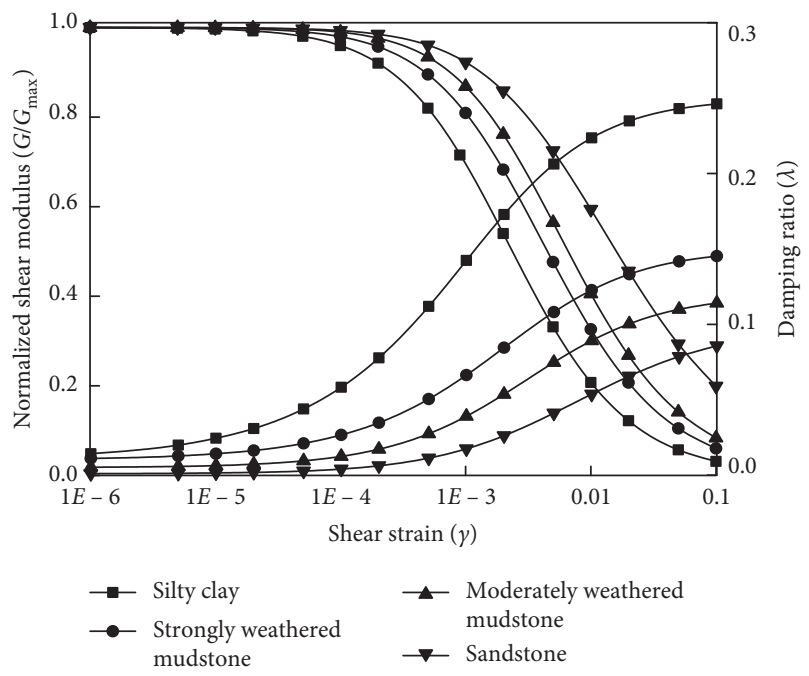

(b)

Figure 5: The stress-strain relationship of the soil and the relationship curve between $G / G_{\max }, \lambda$, and $\gamma$. 
TABLE 4: Constitutive model parameters of the SR site.

\begin{tabular}{lccc}
\hline Soils & $A$ & $B$ & $\gamma_{0}\left(\times 10^{-4}\right)$ \\
\hline Silty clay & 1.202 & 0.457 & 18.09 \\
Strongly weathered mudstone & 1.313 & 0.439 & 30.01 \\
Moderately weathered mudstone & 1.531 & 0.422 & 35.27 \\
Sandstone & 1.727 & 0.351 & 60.42 \\
\hline
\end{tabular}

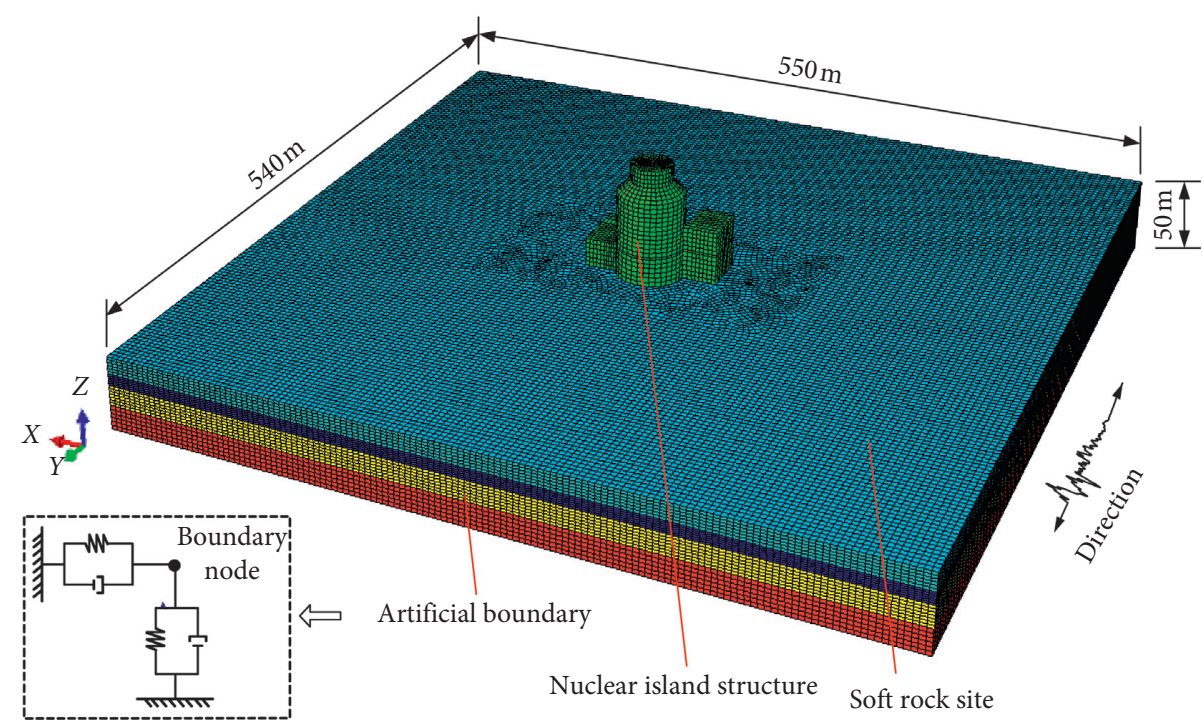

FIGURE 6: Calculation model of the SR foundation-nuclear island structure system.

3.4. Input Ground Motion. There are two methods to determine the input ground motion in site seismic safety evaluation in China, namely, the deterministic method and the probabilistic method. The deterministic method involves characterizing the hypocenter by the seismogenic structure and its largest potential earthquake or the seismotectonic area and its maximum diffuse earthquake. The probabilistic method involves characterizing the hypocenter by the potential seismic source and its seismic activity parameters. Based on these two methods for the site seismic safety evaluation results, it was determined that the SL-1 and SL-2 peak accelerations of the horizontal bedrock ground motion of the proposed nuclear power plant site were $0.10 \mathrm{~g}$ and $0.20 \mathrm{~g}$, respectively. In order to study the impact of the ground motion characteristics on the seismic response of the nuclear island structure, the near-field and far-field actual seismic records were selected as the input bedrock ground motion (Table 5), and each seismic record was amplitudemodulated. The peak accelerations of the input bedrock ground motion were taken as $0.05 \mathrm{~g}, 0.10 \mathrm{~g}$, and $0.20 \mathrm{~g}$. The ground motion was input along the direction of the nuclear island structure with a slightly weaker stiffness (i.e., the translation direction of the first-order mode, as shown in Figure 3). The acceleration time history and the Fourier spectrum of the original seismic records are shown in Figure 7.

\section{Calculation Results and Analysis}

\subsection{Floor Acceleration}

4.1.1. Acceleration Time History. In this research, the observation points were set at the representative locations of $\mathrm{SB}, \mathrm{SCV}$, CIS, and $\mathrm{AB}$ of the nuclear island structure (Figure 8). It was found that the acceleration time history curves were similar at different observation points, but the peak accelerations were different. Figure 8 presents the acceleration time history curve of each observation point when the input ground motion intensity was $0.20 \mathrm{~g}$. It can be seen from the figure that the acceleration response of each observation point showed a magnification effect in a different degree because of the spatial structure characteristics of the nuclear island and the spectrum characteristics of the input ground motion. Furthermore, due to the amplifying effect of the SR foundation and the high-frequency filtering effect, the acceleration response of the nuclear island structure under the impact of the near-field MZQP wave was smaller than that under the impact of the far-field SUCHIL wave. The peak acceleration of each observation point was closely related to its height. Specifically, the peak acceleration at the top of the SB (the highest point of the entire nuclear island structure) was the largest, followed by the peak acceleration at the top of the SCV, while the peak acceleration 
TABLE 5: Input bedrock ground motion information.

\begin{tabular}{|c|c|c|c|c|c|c|c|c|}
\hline Earthquake name & $\begin{array}{l}\text { Seismic } \\
\text { wave }\end{array}$ & Station & $\begin{array}{c}\text { Fault } \\
\text { distance } \\
(\mathrm{km})\end{array}$ & Direction & $\begin{array}{l}\text { Predominant } \\
\text { period (s) }\end{array}$ & $\begin{array}{l}\text { PGA } \\
(\mathrm{g})\end{array}$ & $\begin{array}{c}\text { Arias } \\
\text { intensity }(\mathrm{m} / \\
\mathrm{s})\end{array}$ & $\begin{array}{c}\text { Significant } \\
\text { duration } D_{5-95}(\mathrm{~s})\end{array}$ \\
\hline $\begin{array}{l}\text { Wenchuan, China, } \\
\text { 2008, Ms8.0 }\end{array}$ & $\begin{array}{c}\text { MZQP } \\
\text { wave }\end{array}$ & $51 \mathrm{MZQ}$ & $2.0 \mathrm{~km}$ & EW & 0.10 & 0.841 & 11.622 & 30.065 \\
\hline $\begin{array}{l}\text { Michoacan, Mexico, } \\
\text { 1985, Ms8.1 }\end{array}$ & $\begin{array}{l}\text { SUCHIL } \\
\text { wave }\end{array}$ & SUCHIL & 226.4 & NS & 0.35 & 0.078 & 0.0657 & 20.48 \\
\hline
\end{tabular}

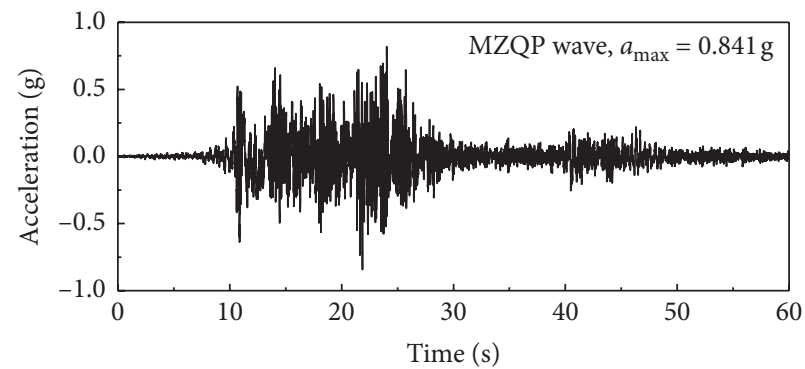

(a)

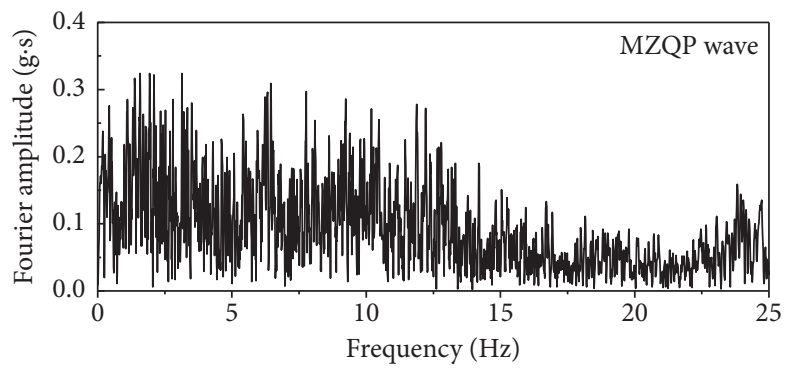

(c)

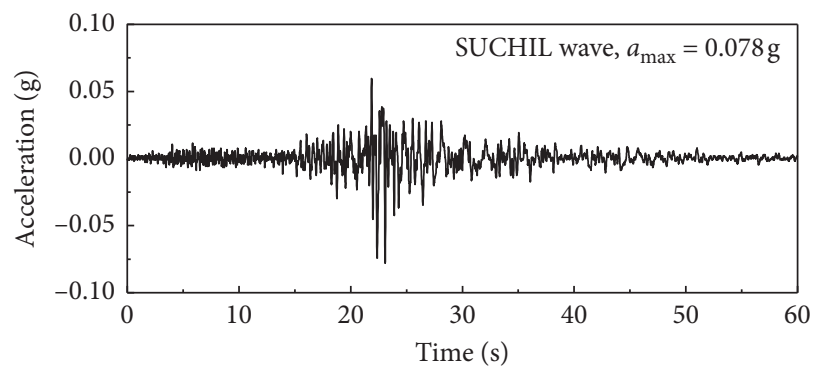

(b)

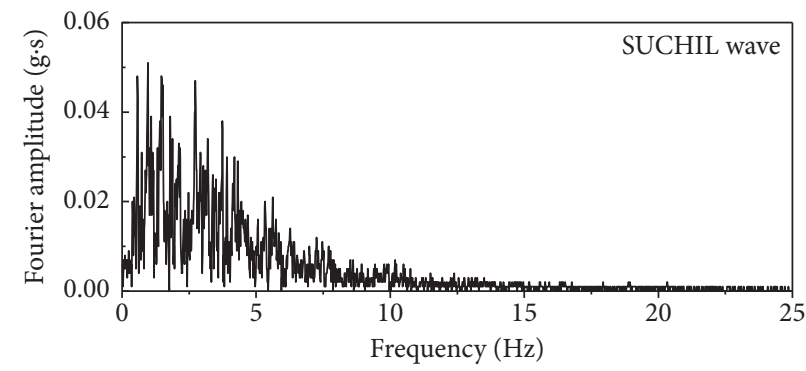

(d)

Figure 7: Acceleration time history and Fourier spectrum of input bedrock ground motion.
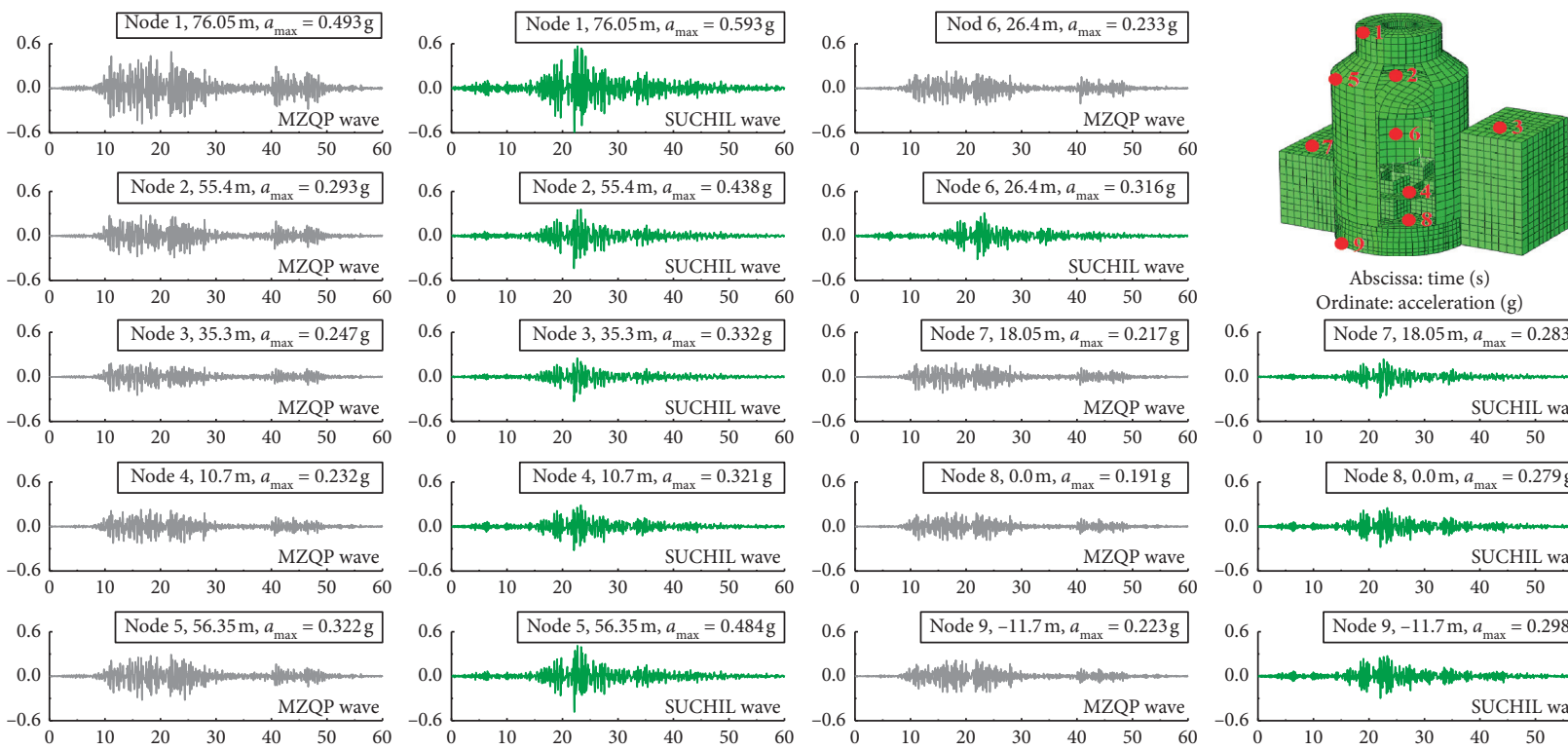

Ordinate: acceleration $(\mathrm{g})$
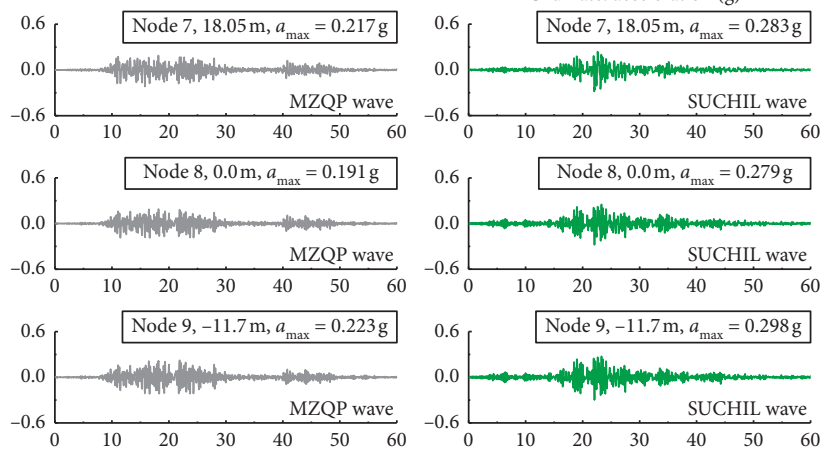

FIGURE 8: Acceleration response of the nuclear island structure observation points. 
at the top of the $\mathrm{AB}$ was slightly larger than that of the CIS. Therefore, as a Class I seismic structure, the passive cooling water tank was set on the top of the SB, which was an important focus during the seismic analysis of the nuclear island structure.

4.1.2. Peak Floor Acceleration (PFA). Figure 9 shows the changing trend of the PFA of the nuclear island structure as a function of the height. It can be seen from the figure that, with the increase of the height, the PFAs of the SB, SCV, AB, and CIS generally increased to varying degrees. Specifically, the PFA of the SB increased rapidly, especially at the higher part of the structure, while the PFA of the lower part of the SB was slightly smaller than that of the base floor, which was mainly related to the larger stiffness of the base floor and the complicated structure of the $\mathrm{AB}$ around the SB. These characteristics were beneficial to the seismic resistance of the pipelines and equipment inside the SCV, and the same law was also reported by [9]. Additionally, the centralized mass rod model was used to simplify the bottom constraint of the nuclear island foundation. No reduction was observed in the peak acceleration, but, generally, this did not affect the dynamic characteristics of the nuclear island structure system $[34,35]$. The SCV had a relatively larger stiffness and larger height, and its PFA showed an increasing trend with height, while the PFA of the AB and the CIS only increased slightly.

With the increase of the input ground motion intensity, the PFA of the nuclear island structure also increased. Because the SR site filtered the MZQP wave with richer medium-high frequencies and it amplified the SUCHIL wave with more developed low frequencies, the increasing amplitude of the PFA under the input of the SUCHIL wave was larger than that under the input of the MZQP wave. Therefore, the PFA of the nuclear island structure for nonlinear SR sites was affected by the spectrum characteristics of the input ground motion. That is, with the enrichment of the medium-high-frequency components in the input ground motion, the increasing amplitude of the PFA of the nuclear island structure decreased with the increase of the input ground motion intensity.

4.1.3. Amplification Factor (AF) of the PFA. In order to better analyze the acceleration response of the nuclear island structure, the AFs of the PFA relative to the bedrock and the basemat were calculated with the following equations:

$$
\begin{aligned}
& \mathrm{AF}_{\text {bedrock }}=\frac{\mathrm{PA}_{\mathrm{NIS}}}{\mathrm{PA}_{\mathrm{BGM}}} \\
& \mathrm{AF}_{\text {basemat }}=\frac{\mathrm{PA}_{\mathrm{NIS}}}{\mathrm{PA}_{\mathrm{NIB}}},
\end{aligned}
$$

where $\mathrm{PA}_{\mathrm{NIS}}$ is the PFA of the nuclear island structure, $\mathrm{PA}_{\mathrm{BGM}}$ is the peak acceleration of the bedrock ground motion, and $\mathrm{PA}_{\mathrm{NIB}}$ is the peak acceleration of the nuclear island basemat.
Figure 10 shows the distributions of $\mathrm{AF}_{\text {bedrock }}$ and $\mathrm{AF}_{\text {basemat }}$ at the top of the $\mathrm{SB}, \mathrm{SCV}, \mathrm{AB}$, and CIS. It can be seen from the figure that as the input ground motion intensity increased, the SR site gradually entered a nonlinear state, and $\mathrm{AF}_{\text {bedrock }}$ shows a decreasing trend. The decrease of $\mathrm{AF}_{\text {bedrock }}$ was more obvious for the input far-field SUCHIL wave. $\mathrm{AF}_{\text {basemat }}$ showed an increasing trend, and $\mathrm{AF}_{\text {basemat }}$ increased faster when the near-field MZQP wave was input. Because it was affected by the characteristics of the ground motion spectrum, the AF of the PFA of the nuclear island structure under the input of the far-field ground motion was generally larger than that under the input of the near-field ground motion. In view of the spatial difference of the nuclear island structure, the AF of the PFA at the top of the SB was the largest, followed by that of the SCV, while the AF of the PFA of the CIS was slightly larger than that of the $A B$.

The change characteristics of the AF of the PFA with the increase of the input ground motion intensity indicated that the SR site had gradually entered a nonlinear state. The Niigata-ken Chuetsu-oki Earthquake (2007) and Suruga Bay Earthquake (2009) in Japan both appeared to have nonlinear behaviors for the SR sites of nuclear power plants [36-38]. Combined with the AF of the relative basemat, it was found that the amplification effect of the nuclear island structure caused by near-field ground motion mainly depended on the characteristics of the nuclear island structure itself, while the amplification effect of the nuclear island structure caused by far-field ground motion mainly depended on the propagation of the bedrock ground motion through the nonlinear SR site to the nuclear island foundation bottom. Compared with the near-field high-frequency ground motion, the farfield low-frequency ground motion was more destructive to the nuclear island structure. Therefore, during the SSI analysis of a nuclear island structure, it is necessary to consider not only the nonlinear characteristics of the soil but also the influence of the near-far field spectral characteristics.

4.2. Floor Response Spectrum (FRS). The acceleration response spectrum reflects the influence of the input ground motion on the dynamic characteristics of a nuclear island structure by comprehensively considering the input ground motion, the dynamic characteristics of the nuclear island structure, and the relationship between the two. It is an important indicator for the seismic response characteristics of a nuclear island structure, and it is an important part of seismic performance analysis. In this research, the acceleration response spectrum $S_{a}(T)$ of the nuclear island structure was normalized using equation (5), and the dimensionless dynamic coefficient $\beta$ spectrum was obtained as the FRS of the nuclear island structure.

$$
\beta(T)=\frac{S_{a}(T)}{\text { PFA }}
$$

where $\beta(T)$ is the dynamic coefficient and $S_{a}(T)$ is the acceleration response spectrum of the nuclear island structure. 

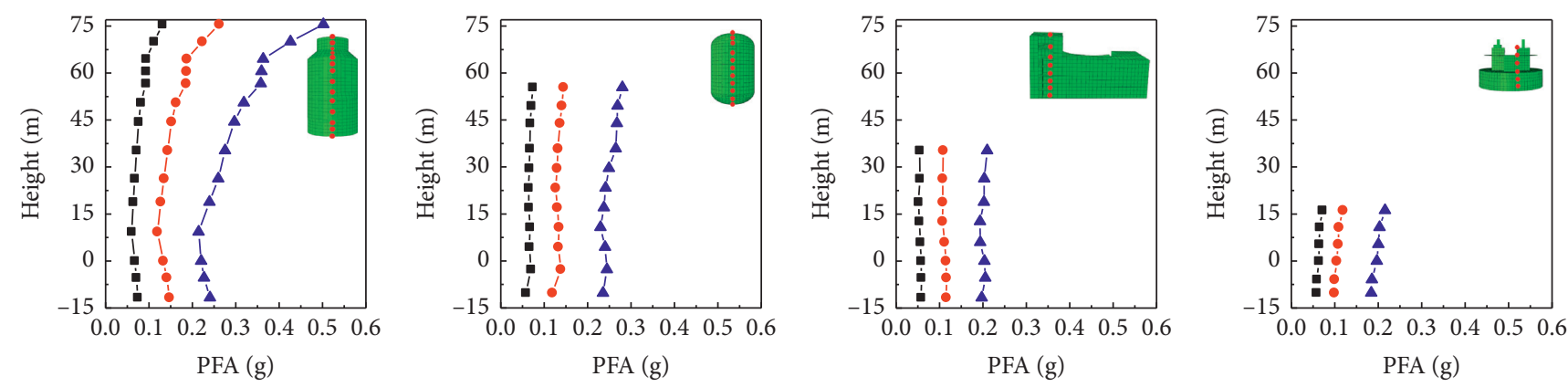

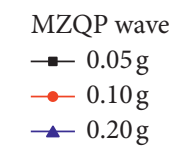

(a)

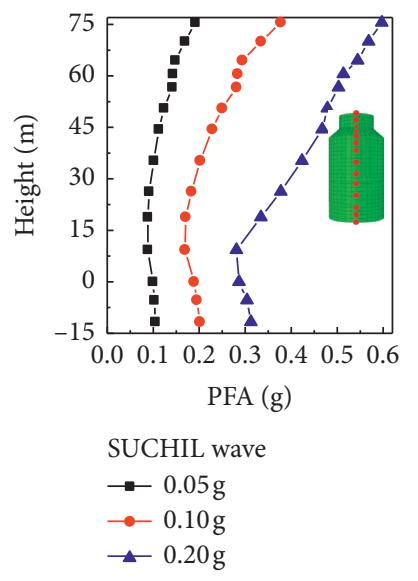

(e)

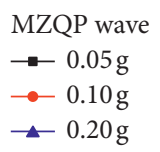

(b)

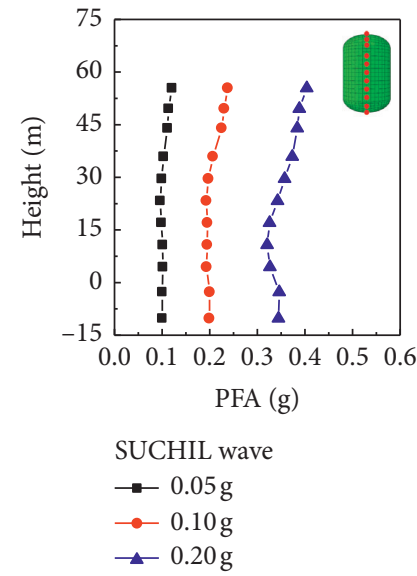

(f)

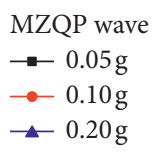

(c)

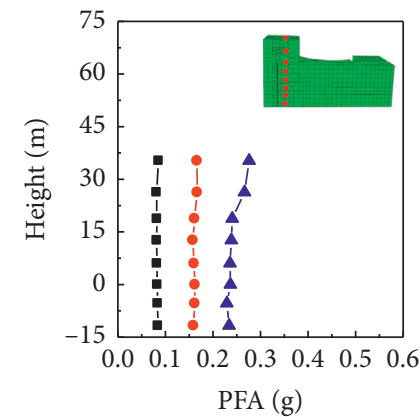

SUCHIL wave

$$
\begin{aligned}
& \longrightarrow 0.05 \mathrm{~g} \\
& \longrightarrow 0.10 \mathrm{~g}
\end{aligned}
$$$$
-0.20 \mathrm{~g}
$$

(g)

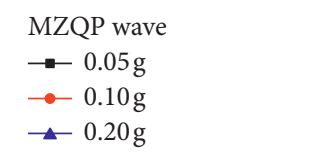

(d)

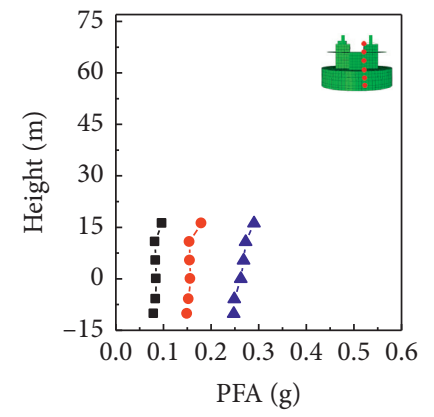

SUCHIL wave

$\rightarrow-0.05 \mathrm{~g}$

$\rightarrow 0.10 \mathrm{~g}$

$\rightarrow 0.20 \mathrm{~g}$

(h)

FIGURE 9: Relationship between the PFA and the height of the nuclear island structure.

Figure 11 shows the FRS of the nuclear island structure observation points with a $5 \%$ damping ratio. It can be seen from the figure that, due to the high-frequency filtering effect of the SR site and the complexity of the nuclear island structure, the bedrock spectrum was larger than the FRS of the nuclear island structure in a short-term period $(<0.2 \mathrm{~s})$, especially for the near-field ground motion. In the mid-term period, the FRS of the nuclear island structure was greater than the bedrock spectrum in the period of $0.2-1.0 \mathrm{~s}$ for the near-field ground motion, and the FRS of nuclear island structure was greater than the bedrock spectrum in the period of $0.2-0.7 \mathrm{~s}$ for the far-field ground motion. In the long-term period, the FRS of the nuclear island structure showed a consistent changing trend, and the difference became smaller and smaller. With the input of the near-field MZQP wave, the FRS of the nuclear island structure moved obviously toward the long-period direction (low-frequency band), while with the input of the far-field SUCHIL wave, the FRS of the nuclear island structure moved slightly toward the short-period direction (high-frequency band). Under the effect of the far-field SUCHIL wave, the peak FRS of the nuclear island structure was relatively large in the period of $0.20-0.40 \mathrm{~s}$, and it showed a double-peak characteristic. Its main peak period was $0.22-0.25 \mathrm{~s}$, and the secondary peak period was about $0.27 \mathrm{~s}$. The peaks were relatively close, and they were close to the basic period of the nuclear island structure $(0.235 \mathrm{~s}$; the corresponding natural frequency was $4.26 \mathrm{~Hz}$ ). Therefore, the nuclear island structure had a relatively stronger seismic response under the effect of the far-field SUCHIL wave. The peak FRS of the nuclear island structure increased with the increase of the input ground motion intensity, but the corresponding predominant period remained essentially unchanged.

Figure 12 shows the changes of the FRS of the SB, SCV, $\mathrm{AB}$, and $\mathrm{CIS}$ with height when the input ground motion intensity was equal to $0.2 \mathrm{~g}$. It can be seen from the figure that the higher the height of the nuclear island structure observation point, the smaller the peak FRS, but the predominant period was essentially the same. Under the effect of the near-field MZQP wave, the FRS of the SB, SCV, AB, and CIS was narrower and more consistent; the peak FRS of the base floor was significantly larger, while the peak FRS of 


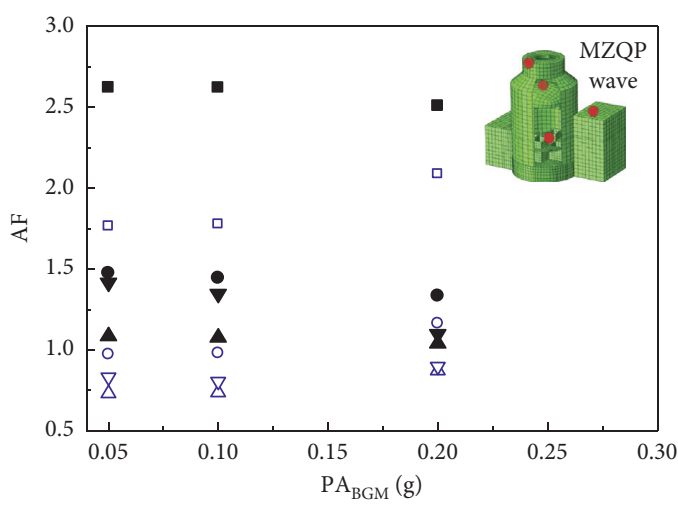

Basemat Bedrock

$\begin{array}{lll}\square & \bullet & \text { SB } \\ \circ & \bullet & \text { SCV } \\ \Delta & \boldsymbol{\Delta} & \text { AB } \\ \nabla & \boldsymbol{\nabla} & \text { CIS }\end{array}$

(a)

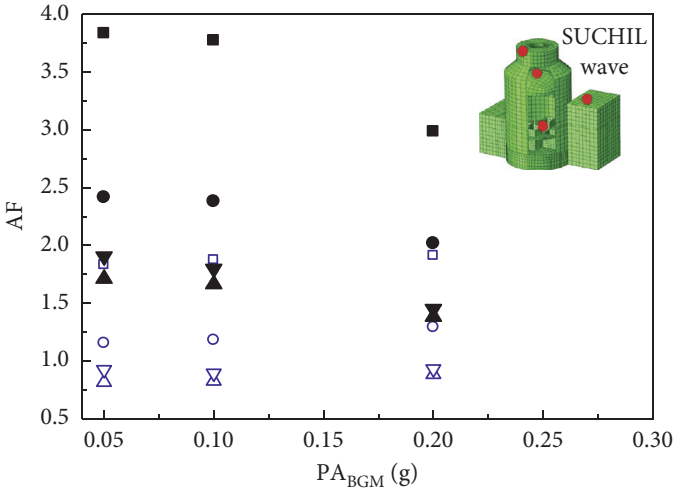

Basemat Bedrock

$\begin{array}{lll}\square & \bullet & \mathrm{SB} \\ \circ & \bullet & \mathrm{SCV} \\ \Delta & \boldsymbol{\Delta} & \mathrm{AB} \\ \nabla & \boldsymbol{\nabla} & \mathrm{CIS}\end{array}$

(b)

Figure 10: AF of the PFA of the nuclear island structure.
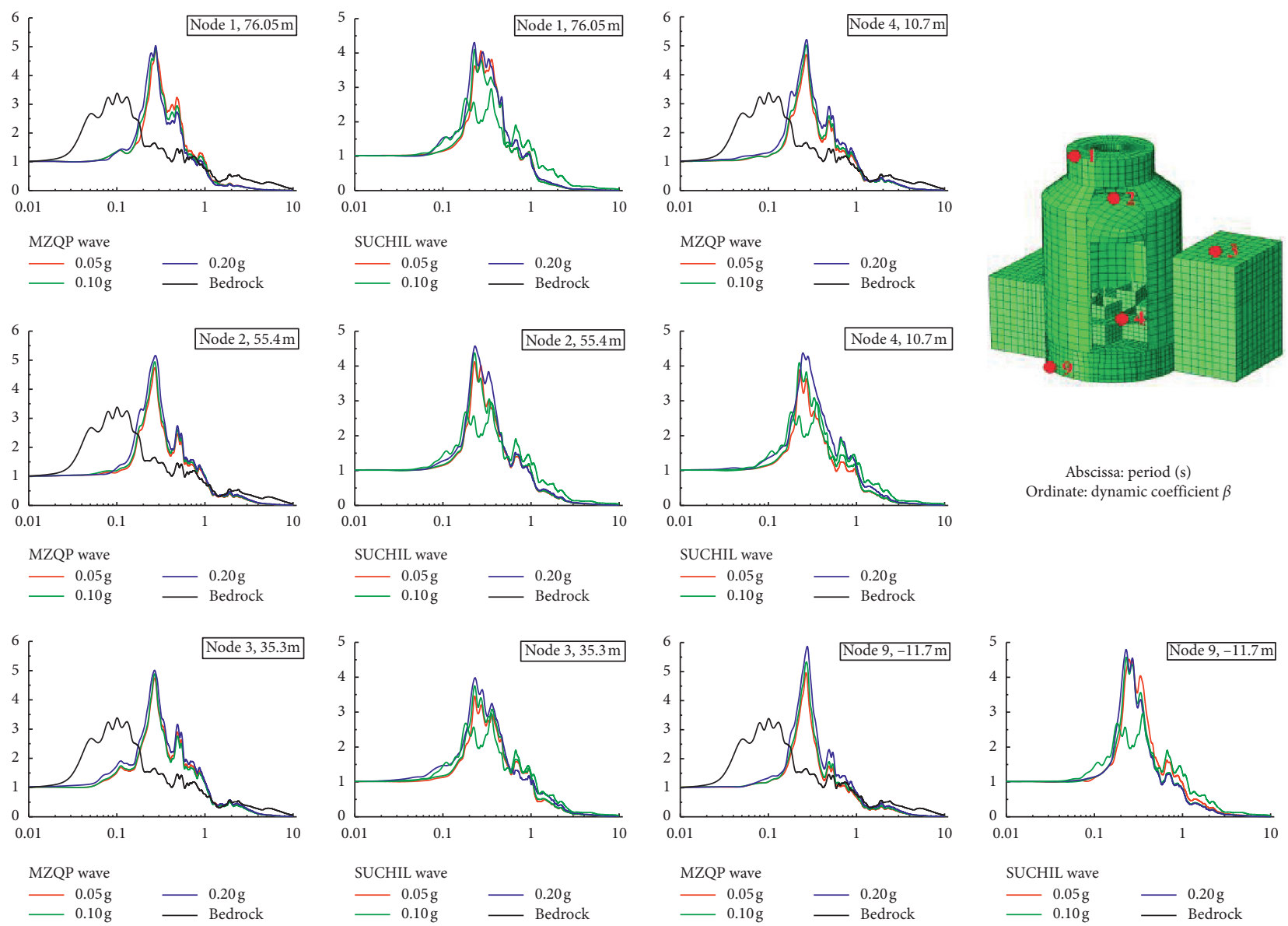

FIGURE 11: FRS of the nuclear island structure observation points.

the other floors had no significant difference. Under the effect of the far-field SUCHIL wave, there were differences in the double-peak spectrum shapes of the FRS of the SB, SCV, $\mathrm{AB}$, and CIS, and the changes in the peak FRSs on different floors were relatively uniform.
4.3. Floor Relative Displacement. The relative displacement of different parts of the nuclear island structure under a seismic load directly reflected the degree of seismic damage to the nuclear island structure. Figure 13 shows the peak floor relative displacement (PFRD) of the nuclear island 


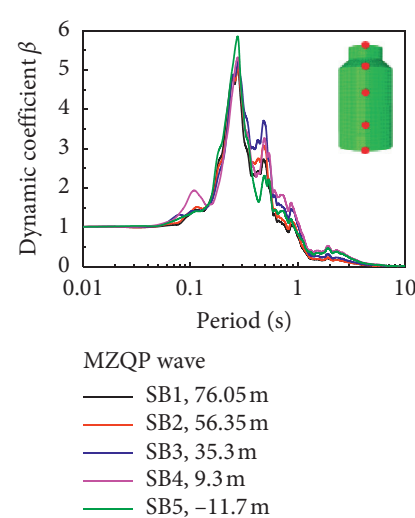

(a)

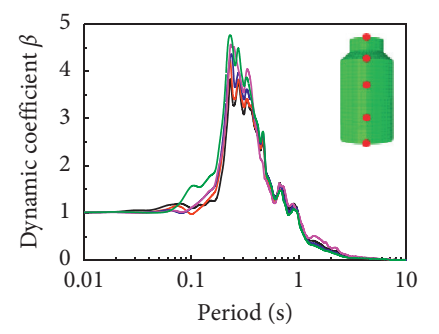

SUCHIL wave

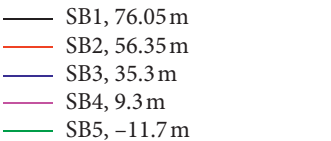

(e)
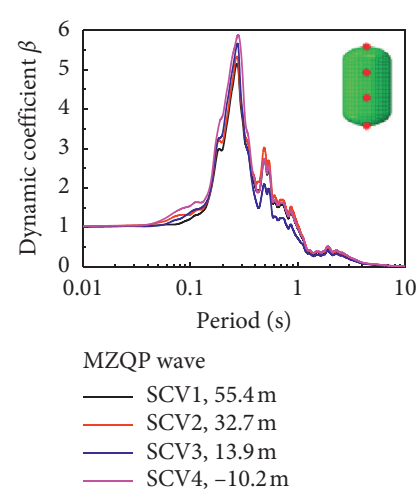

(b)

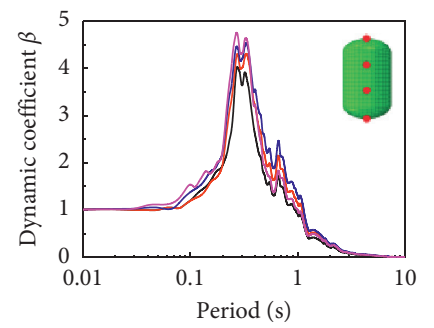

SUCHIL wave

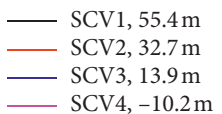

(f)
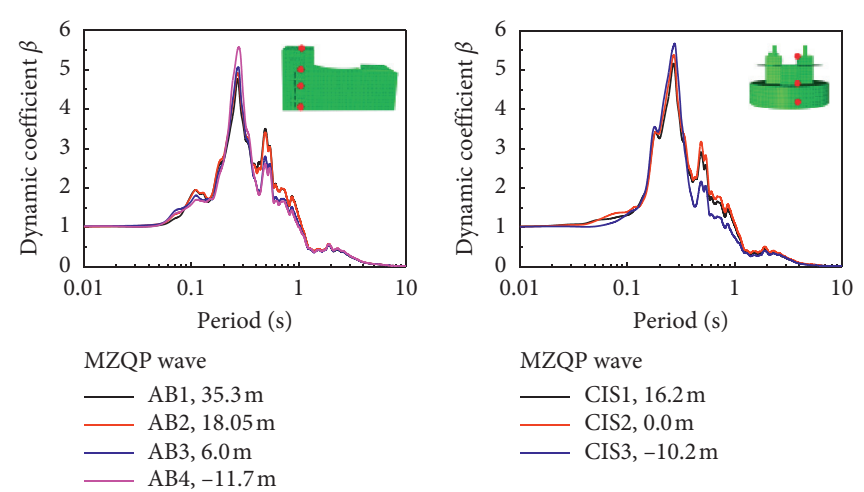

(c)

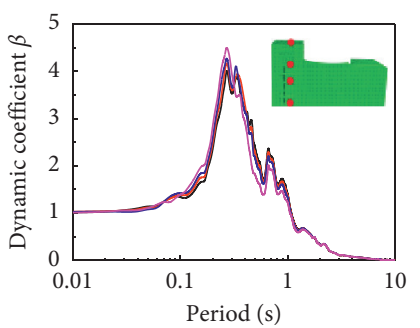

SUCHIL wave

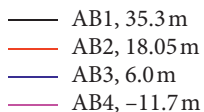

(g) (d)

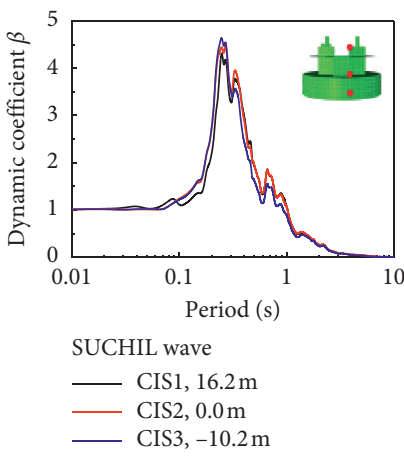

(h)

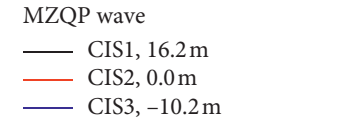

FIgURE 12: Changes of the FRS with the height of the nuclear island structure.

structure, that is, the absolute value of the maximum difference between the horizontal displacement time history of each observation point and the horizontal displacement time history of the basemat. It can be seen that the PFRD at the top of the SB was significantly increased, which may have been caused by the weak whiplash effect at the tapered roof of the SB. This part was installed with the passive cooling water tank, so its seismic load should have been appropriately increased during seismic design. With the increase of the height of the observation point at the SB, SCV, AB, and CIS, the corresponding PFRD showed an increasing trend. Generally, the PFRD of the nuclear island structure under the effect of the far-field SUCHIL wave with richer low frequencies was larger than that under the effect of the nearfield MZQP wave. The top of the SB, which was the highest location of the entire nuclear island structure, had the largest relative displacement. For the SL-2 limiting safe ground motion, that is, when the input ground motion intensity was $0.20 \mathrm{~g}$, the PFRD at the top of the $\mathrm{SB}$ was close to $2.5 \mathrm{~cm}$, which met the deformation requirement of the nuclear island structure for normal use and which had a high safety margin [39].

With the enrichment of the medium-high-frequency components of the input ground motion, the increasing amplitude of the PFRD of the nuclear island structure decreased gradually with the increase of the input ground motion intensity. When the input ground motion intensity increased from $0.05 \mathrm{~g}$ to $0.20 \mathrm{~g}$, the increasing amplitudes of the PFRD of the SB and the SCV were $1.787 \mathrm{~cm}$ and $1.055 \mathrm{~cm}$, respectively, under the effect of SUCHIL wave, and they were $1.658 \mathrm{~cm}$ and $0.933 \mathrm{~cm}$, respectively, under the effect of the MZQP wave with richer medium-high frequencies. Therefore, the relative displacement of the nuclear island structure under the SSI effect was related to the spectral characteristics of the input ground motion. The farfield ground motion with further epicenters and relatively fewer medium-high-frequency components might have caused more serious potential damage to the nuclear island structure.

Regarding the nonlinear effect, from the relative displacement response in Figure 13, it can be seen that the nonlinear relationship was not obvious. This was mainly because although CAP1400 nuclear island site used in this research was a soft rock site, the equivalent shear wave velocity of the site reached $515 \mathrm{~m} / \mathrm{s}$, and the stiffness was even larger. Therefore, although the nonlinear constitutive model was used, the nonlinear effect might not have been obvious in a site with such a large shear wave velocity, which led to the fact that the nonlinear effect in the figure could not be clearly reflected. In addition, according to the site seismic safety assessment report, $0.05 \mathrm{~g}, 0.10 \mathrm{~g}$, and $0.20 \mathrm{~g}$ were selected as the input peak accelerations. However, because the ground motion intensity was not particularly strong, the soft rock site might still have been mainly elastically deformed, 


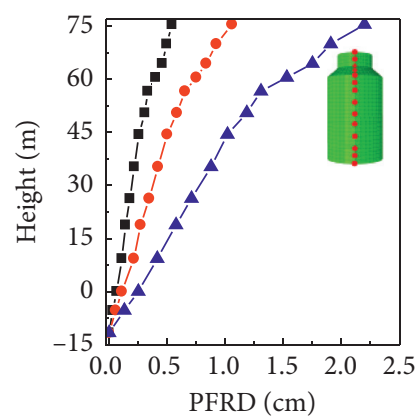

MZQP wave

-. $0.05 \mathrm{~g}$

$-0.10 \mathrm{~g}$

- $\mathbf{- \Delta} 0.20 \mathrm{~g}$

(a)

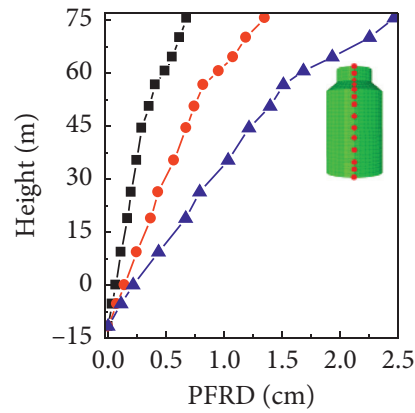

SUCHIL wave

$-0.05 \mathrm{~g}$

$-0.10 \mathrm{~g}$

$-\Delta-0.20 \mathrm{~g}$

(e)

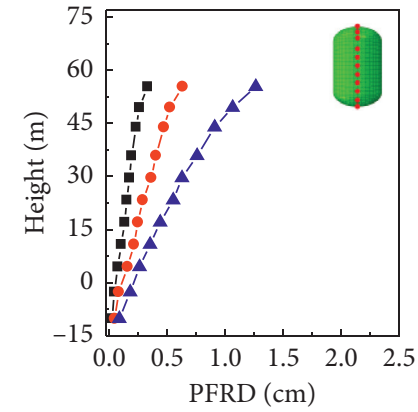

MZQP wave

- - $0.05 \mathrm{~g}$

$-\bullet 0.10 \mathrm{~g}$

$-\mathbf{-}-0.20 \mathrm{~g}$

(b)

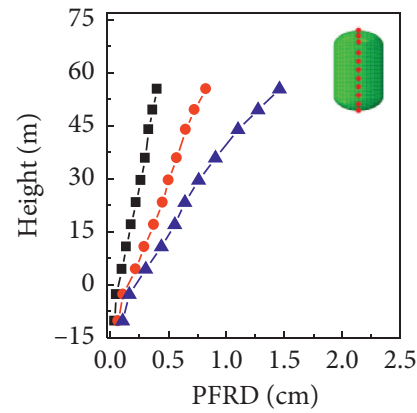

SUCHIL wave

$-\pi-0.05 \mathrm{~g}$

$-\bullet 0.10 \mathrm{~g}$

$-\mathbf{-}-0.20 \mathrm{~g}$

(f)

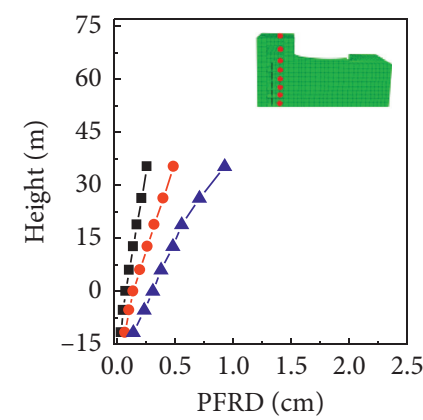

$$
\begin{aligned}
& \text { MZQP wave } \\
& -\mathbf{-}-0.05 \mathrm{~g} \\
& -\bullet-0.10 \mathrm{~g} \\
& -\mathbf{-}-0.20 \mathrm{~g}
\end{aligned}
$$

(c)

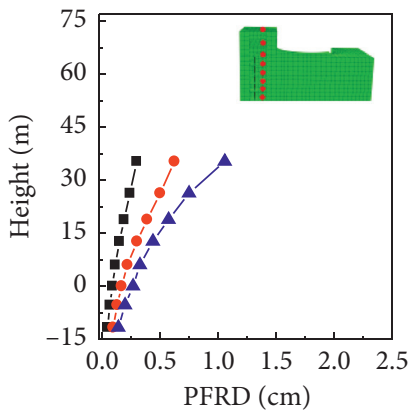

$$
\begin{aligned}
& \text { SUCHIL wave } \\
& -\bullet-0.05 \mathrm{~g} \\
& -\bullet-0.10 \mathrm{~g} \\
& -\mathbf{-}-0.20 \mathrm{~g}
\end{aligned}
$$

(g)

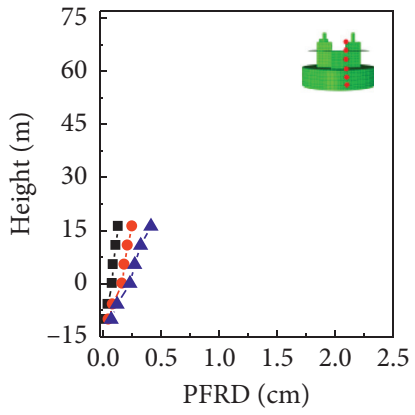

MZQP wave

$-0.05 \mathrm{~g}$

$-\bullet 0.10 \mathrm{~g}$

-4- $0.20 \mathrm{~g}$

(d)

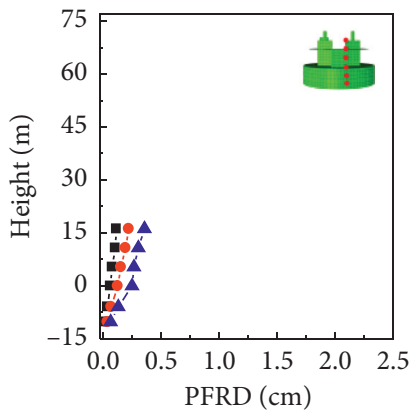

SUCHIL wave

$$
\begin{aligned}
& -0.05 \mathrm{~g} \\
& --0.10 \mathrm{~g}
\end{aligned}
$$$$
-\mathbf{-}-0.20 \mathrm{~g}
$$

(h)

FIGURE 13: Relationship between the PFRD and the height of the nuclear island structure.

resulting in insignificant nonlinear effects. Nonetheless, there are still some reflections on the nonlinear effect in Figures 9 and 10 for the acceleration changes of the nuclear island structure.

\section{Study of the Critical Shear Wave Velocity of Nonbedrock Sites}

Due to the advancement and novelty of CAP1400 nuclear power plant, it will definitely be built more and more frequently in the future. However, the critical shear wave velocity of a nonbedrock site suitable for CAP1400 nuclear island structure has yet to be studied. Therefore, five types of SR stratums measured from the geological survey data of nuclear power plants were selected first in this research. The basic mechanical parameters are shown in Table 6. Based on this, the influence of the shear wave velocity of the SR site on the PFA, PFRD, and FRS of CAP1400 nuclear island structure was mainly investigated when the input peak bedrock acceleration was $0.2 \mathrm{~g}$. Then the recommended critical shear wave velocity of nonbedrock sites for CAP1400 nuclear island structure was obtained, which provided a necessary reference basis for the site selection and seismic analysis of CAP1400 nuclear power plants.
5.1. Influence of Shear Wave Velocity on PFA of Nuclear Island Structure. Figure 14 shows the changes of the PFA of the nuclear island structure with the shear wave velocity of the SR site at four observation points. It can be seen from the figure that, with the increase of the shear wave velocity, the PFA at each observation point increased accordingly, that is, the PFA of the nuclear island structure built on a harder rock foundation was larger. The PFA at the top of the AB and the CIS was significantly smaller than that of the $\mathrm{SB}$ and the CSV, which had a larger height. The PFA of the CIS was the smallest due to the low height of the CIS observation point and the constraints from the SB and the SCV. It could be seen that the internal structure supporting the nuclear power equipment and the pipelines had high seismic safety. In seismic design, it is recommended that more attention should be paid to the SB and SCV where the earthquake response is more severe, and appropriate seismic measures should be taken.

5.2. Influence of Shear Wave Velocity on PFRD of Nuclear Island Structure. The influence of the shear wave velocity of the SR site on PFRD of the nuclear island structure is illustrated in Figure 15. It can be found from the figure that, 
TABLE 6: Basic mechanical parameters for different SR sites.

\begin{tabular}{lccccc}
\hline \multirow{2}{*}{ Type } & Density $\left(\mathrm{kg} / \mathrm{m}^{3}\right)$ & Shear wave velocity $(\mathrm{m} / \mathrm{s})$ & Predominant period $(\mathrm{s})$ & \multicolumn{3}{c}{ Constitutive model parameters } \\
& & & & $\gamma_{0}\left(\times 10^{-4}\right)$ \\
\hline Site 1 & 1960 & 395 & 0.506 & 1.11 & 0.42 \\
Site 2 & 2070 & 532 & 0.376 & 1.24 & 0.39 \\
Site 3 & 2140 & 720 & 0.278 & 1.39 & 0.36 \\
Site 4 & 2310 & 915 & 0.219 & 1.45 & 0.35 \\
Site 5 & 2350 & 1099 & 0.182 & 1.45 & 38.1 \\
\hline
\end{tabular}

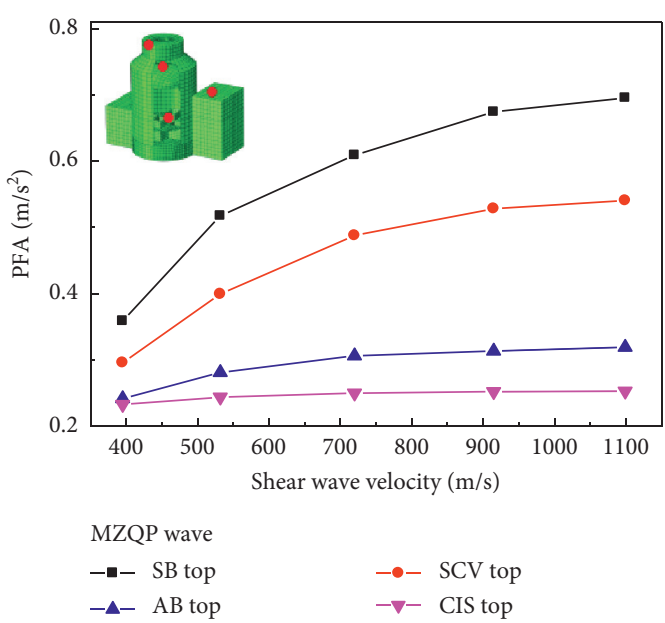

(a)

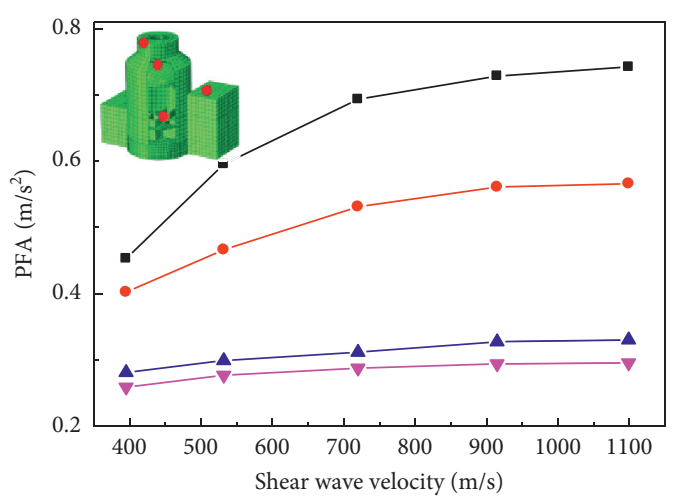

SUCHIL wave

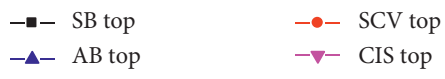

(b)

FIgURE 14: Relationship between the shear wave velocity of the SR site and the PFA of the nuclear island structure.

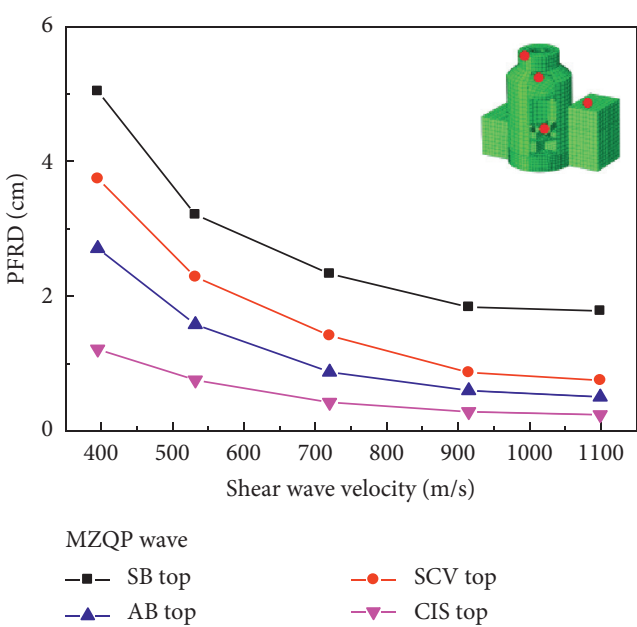

(a)

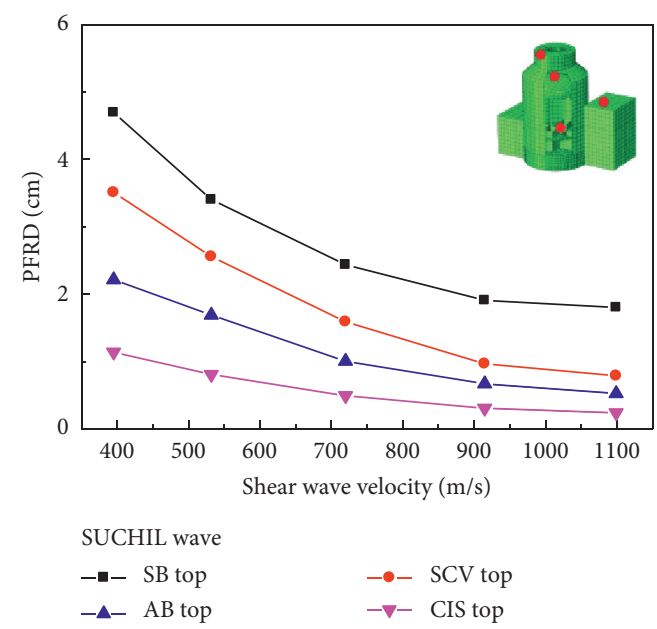

(b)

FIGURE 15: Relationship between the shear wave velocity of the SR site and the PFRD of the nuclear island structure.

with the increase of the shear wave velocity of the SR site, the PFRD at each observation point of the nuclear island structure gradually decreased, but when the shear wave velocity exceeded $915 \mathrm{~m} / \mathrm{s}$, the reduction of the PFRD tended to stabilize, especially under the input of the near-field MZQP wave. When the shear wave velocity was $395 \mathrm{~m} / \mathrm{s}$ (extreme SR site), the PFRD at the top of the SB reached the largest values $(5.04 \mathrm{~cm}$ and $4.70 \mathrm{~cm})$ under the input of the MZQP wave and the SUCHIL wave. Although the stiffness of the nuclear island structure was reduced, it still maintained a good overall performance and met the deformation requirement for the seismic performance of a nuclear island 


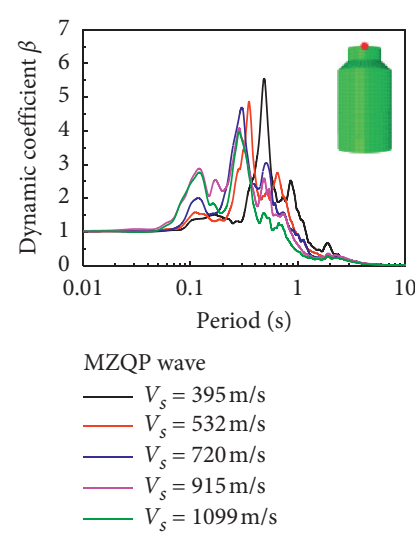

(a)
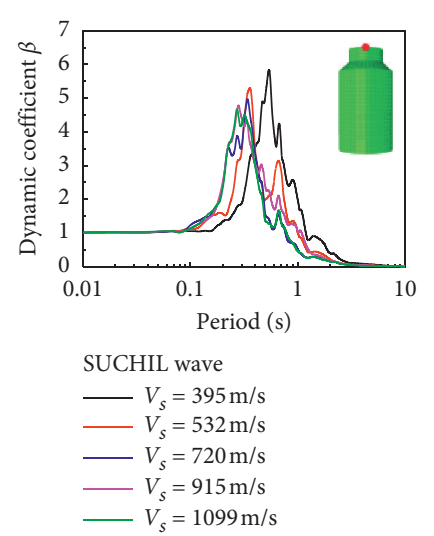

(b)
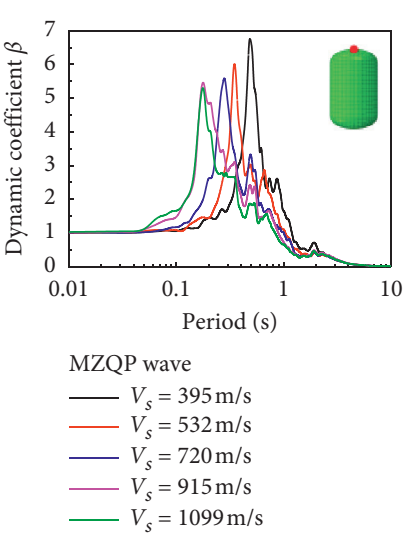

(c)
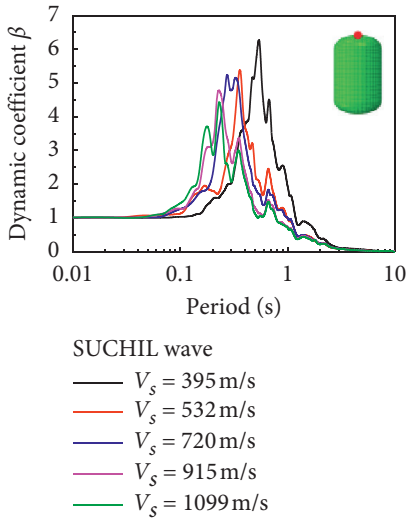

(d)

Figure 16: FRS at the top of the SB and SCV.

TABle 7: PFA and PFRD at the top of the SB.

\begin{tabular}{lccccccccc}
\hline Case & $\begin{array}{c}V_{s} \\
(\mathrm{~m} / \mathrm{s})\end{array}$ & $\begin{array}{c}\text { PFA } a_{i} \\
(\mathrm{~g})\end{array}$ & $\begin{array}{c}\text { MZQP wave } \\
\left(a_{V}-a_{i} / a_{V}\right)(\%)\end{array}$ & $\begin{array}{c}\text { PFRD } d_{i} \\
(\mathrm{~cm})\end{array}$ & $\left(\left|d_{V}-d_{i}\right| / d_{V}\right)(\%)$ & $\begin{array}{c}\text { PFA } a_{i} \\
(\mathrm{~g})\end{array}$ & \multicolumn{3}{c}{ SUCHIL wave } \\
$\left(a_{V}-a_{i} / a_{V}\right)(\%)$ & $\begin{array}{c}\text { PFRD } d_{i} \\
(\mathrm{~cm})\end{array}$ & $\left(\left|d_{V}-d_{i}\right| / d_{V}\right)(\%)$ \\
\hline I & 1099 & 0.695 & 5.47 & 1.781 & 10.35 & 0.742 & 6.74 & 1.653 & 6.44 \\
II & 1150 & 0.712 & 3.18 & 1.727 & 7.00 & 0.752 & 5.41 & 1.580 & 1.74 \\
III & 1200 & 0.721 & 2.01 & 1.649 & 2.17 & 0.761 & 4.38 & 1.562 & 0.58 \\
IV & 1250 & 0.733 & 0.37 & 1.623 & 0.56 & 0.787 & 1.03 & 1.556 \\
V & 1300 & 0.735 & - & 1.614 & - & 0.795 & - & 1.553 & - \\
\hline
\end{tabular}

structure. The PFRD of the nuclear island structure under the effect of the SUCHIL wave was slightly larger than that under the effect of the MZQP wave except for the fact that the shear wave velocity was $395 \mathrm{~m} / \mathrm{s}$. This suggested that the CAP1400 nuclear island structure-foundation system was more sensitive to the near-field wave uniform spectrum ground motion at an extreme SR site. Therefore, when the site of a nuclear power plant is selected at a site with a relatively low shear wave velocity (e.g., sand), special attention should be paid to the influence of near-site earthquake motion.

\subsection{Influence of Shear Wave Velocity on FRS of Nuclear Island} Structure. Figure 16 shows the FRS at the top of the SB and the SCV with the change of the shear wave velocity of the SR site. It can be seen from the figure that, with the increase of the foundation stiffness of the SR site, the peak FRS of the nuclear island structure showed a decreasing trend, and the predominant period showed a decreasing trend, that is, moving toward the low-period (high frequency) direction. In the short-term period ( $T<0.3 \mathrm{~s})$, the FRS on a harder SR site was larger; in the mid-term period (0.3-2 s), the FRS on a softer SR site gradually became larger, especially when the shear wave velocity was smaller. In the long-term period ( $T>2 \mathrm{~s}$ ), the FRS became extremely small, and the influence of the site hardness was no longer significant. This showed that the influence of the shear wave velocity on the nuclear island structural system was mainly reflected in the higher frequency band of the floor response spectrum $(T<0.6 \mathrm{~s})$, and the influence on the low frequency band was small.

As the shear wave velocity of the SR site increased, the shape of the FRS gradually changed from a single peak to a double peak under the input of the far-field SUCHIL wave, while the shape of the FRS remained essentially unchanged under the input of the near-field MZQP wave. The peak FRS of the SB under the effect of the near-field MZQP wave was smaller than that under the effect of the far-field SUCHIL wave, while the peak FRS of SCV showed an opposite trend. This suggested that the island structure and the spectrum characteristics of the input ground motion had very complex effects on the seismic response of the nuclear island at the SR sites. When the shear wave velocity remained unchanged, especially when the shear wave velocity was relatively small, the predominant period of the FRS under the input of the far-field SUCHIL wave was greater than that under the input of the far-field MZQP wave. As the shear wave velocity increased, the FRS of the nuclear island structure under the input of the far-field SUCHIL wave moved faster toward the low-period (high frequency) direction, indicating that the far-field ground motion might have a greater impact on the nuclear island structure.

5.4. Analysis and Suggestion for Critical Shear Wave Velocity on Nonbedrock Sites. Both China's "Code for Seismic Design of Nuclear Power Plants” (GB50267-97) and the US AP1000 Standard Design Guideline point out that SSI must be 
TABLE 8: PFA and PFRD at the top of the SCV.

\begin{tabular}{|c|c|c|c|c|c|c|c|c|c|}
\hline \multirow[b]{2}{*}{ Case } & \multirow{2}{*}{$\begin{array}{c}V_{s} \\
(\mathrm{~m} / \mathrm{s})\end{array}$} & \multicolumn{4}{|c|}{ MZQP wave } & \multicolumn{4}{|c|}{ SUCHIL wave } \\
\hline & & $\begin{array}{l}\mathrm{PFA} a_{i} \\
\quad(\mathrm{~g})\end{array}$ & $\left(a_{V}-a_{i} / a_{V}\right)(\%)$ & $\begin{array}{l}\mathrm{PFRD} d_{i} \\
\quad(\mathrm{~cm})\end{array}$ & $\left(\left|d_{V}-d_{i}\right| / d_{V}\right)(\%)$ & $\begin{array}{l}\mathrm{PFA} a_{i} \\
\quad(\mathrm{~g})\end{array}$ & $\left(a_{V}-a_{i} / a_{V}\right)(\%)$ & $\begin{array}{l}\mathrm{PFRD} d_{i} \\
\quad(\mathrm{~cm})\end{array}$ & $\left(\left|d_{V}-d_{i}\right| / d_{V}\right)(\%)$ \\
\hline I & 1099 & 0.540 & 7.49 & 1.036 & 5.82 & 0.566 & 4.51 & 0.749 & 12.80 \\
\hline II & 1150 & 0.548 & 6.10 & 1.021 & 4.29 & 0.580 & 2.03 & 0.716 & 7.83 \\
\hline III & 1200 & 0.560 & 4.14 & 1.004 & 2.55 & 0.586 & 1.14 & 0.690 & 3.92 \\
\hline IV & 1250 & 0.579 & 0.82 & 0.988 & 0.92 & 0.590 & 0.40 & 0.675 & 1.66 \\
\hline $\mathrm{V}$ & 1300 & 0.584 & - & 0.979 & - & 0.592 & - & 0.664 & - \\
\hline
\end{tabular}

considered when the site shear wave velocity is less than $1100 \mathrm{~m} / \mathrm{s}$. The results of this research showed that when the shear wave velocity of the SR site gradually increased to $1099 \mathrm{~m} / \mathrm{s}$, the changes of the PFA and the PFRD of the nuclear island structure both tended to stabilize. However, this was not enough to determine the critical shear wave velocity of the nonbedrock sites for CAP1400 nuclear island structure. Therefore, in this research, $1150 \mathrm{~m} / \mathrm{s}, 1200 \mathrm{~m} / \mathrm{s}$, $1250 \mathrm{~m} / \mathrm{s}$, and $1300 \mathrm{~m} / \mathrm{s}$ were taken as the site shear wave velocities to obtain the PFA and the PFRD at the top of the SB and the SCV, as shown in Tables 7 and 8. It can be seen from the tables that when the shear wave velocity exceeded $1099 \mathrm{~m} / \mathrm{s}$, the changes of the PFA and the PFRD at the top of the SB and the SCV obviously slowed down. Compared with case $V$, the maximum change rates of the PFA and the PFRD were $7.49 \%$ and $12.80 \%$, respectively. Specifically, when the shear wave velocity was $1250 \mathrm{~m} / \mathrm{s}$, the minimum change rate was $0.19 \%$, the maximum change rate was only $1.66 \%$, and the effect of the shear wave velocity was negligible. Therefore, when the site shear wave velocity was greater than $1250 \mathrm{~m} / \mathrm{s}$, the shear wave velocity had a very minimal effect on the seismic response of CAP1400 nuclear island structure-foundation system, and SSI could be ignored during the seismic analysis of the nuclear island structure. It is worth mentioning that the shear wave velocity here is the average value in the depth range of $0-50 \mathrm{~m}$.

\section{Conclusions}

The seismic performance of a nuclear island structure is one of the core concepts in the CAP 1400 project safety evaluation, and the seismic response characteristics of the nuclear island structures on SR sites are the focus of the current research related to nuclear power plant seismic and site selection, specifically. Based on the actual SR site of a nuclear power plant, in this research, the nonlinear characteristics of an SR site and SSI were considered. In addition, a threedimensional refined finite element model of CAP1400 nuclear island structure on the SR site was established, and the seismic response characteristics of CAP1400 nuclear island structure on the SR site with near-far field ground motions were studied. By analyzing the influence of the shear wave velocity of the SR site on the seismic response characteristics of the nuclear island structure, the recommended critical shear wave velocity of nonbedrock sites for CAP1400 nuclear island structure was proposed. The conclusions and recommendations are as follows.
(1) As the input ground motion intensity increased, the SR site gradually entered a nonlinear state, and the nonlinear seismic effect of the SR foundation-nuclear island structure system intensified accordingly. Furthermore, the acceleration and relative displacement response of the nuclear island structure also increased with the input ground motion intensity, but the increasing amplitude of the PFA and the PFRD was reduced. The PFA and the PFRD at the top of the SB, where the cooling water tank was located, were the largest, and this might be considered to increase the seismic load at this location appropriately during seismic design.

(2) The peak FRS of the nuclear island structure decreased with the enrichment of the high-frequency components in the input ground motion, and it moved toward the long-period and short-period directions with the near-field and far-field ground motions, respectively. The peak FRS decreased with the increase of the height of the observation point, and the predominant period remains essentially unchanged. Additionally, the peak FRS decreased with the increase of the shear wave velocity of the SR site, and it moved toward the low-period direction.

(3) The amplification effect of the nuclear island structure caused by the near-field and far-field ground motions mainly depended on the characteristics of the nuclear island structure itself and the propagation of the bedrock ground motion through the nonlinear SR site to the nuclear island foundation bottom. Due to the amplification effect of the SR site and the high-frequency filtering effect, the far-field ground motion was more destructive to the nuclear island structure.

(4) The critical shear wave velocity of the nonbedrock sites for CAP1400 nuclear island structure was $1250 \mathrm{~m} / \mathrm{s}$, that is, for the seismic analysis of CAP1400 nuclear island structure, when the site shear wave velocity was greater than $1250 \mathrm{~m} / \mathrm{s}$, SSI could be ignored during seismic design.

\section{Data Availability}

The data used to support the findings of this study are available from the corresponding author upon request. 


\section{Conflicts of Interest}

The authors declare that they have no conflicts of interest.

\section{Acknowledgments}

This work was supported by the National Key R\&D Program of China (2018YFC1504301), the National Natural Science Foundation of China (51708273), and National Grand Science and Technology Special Project of China (2013ZX06002001-9).

\section{References}

[1] L. Tuñón-Sanjur, R. S. Orr, S. Tinic, and D. P. Ruiz, "Finite element modeling of the AP1000 nuclear island for seismic analyses at generic soil and rock sites," Nuclear Engineering and Design, vol. 237, no. 12-13, pp. 1474-1485, 2007.

[2] X. Wang and Y. Lu, "Optimization of the cross section area on the meridian surface of the 1400-MW canned nuclear coolant pump based on a new medial axial transform design method," Annals of Nuclear Energy, vol. 115, pp. 466-479, 2018.

[3] S. C. Hwang and J. B. Jang, "The study on seismic stability evaluation model for rock foundation of nuclear power plant," Journal of the Earthquake Engineering Society of Korea, vol. 9, no. 4, pp. 1-10, 2005.

[4] T. J. Katona, E. Győri, and L. Tóth, "Applicability of pseudoprobabilistic method of liquefaction hazard assessment for nuclear power plants at diffuse seismicity sites," Science and Technology of Nuclear Installations, vol. 2019, Article ID 5010814, 10 pages, 2019.

[5] I. J. Tromans, G. Aldama-Bustos, J. Douglas et al., "Probabilistic seismic hazard assessment for a new-build nuclear power plant site in the UK," Bulletin of Earthquake Engineering, vol. 17, no. 1, pp. 1-36, 2019.

[6] M. Ashiquzzaman and K.-J. Hong, "Simplified model of soilstructure interaction for seismically isolated containment buildings in nuclear power plant," Structures, vol. 10, pp. 209-218, 2017.

[7] S. Kwag, B. Ju, and W. Jung, "Beneficial and detrimental effects of soil-structure interaction on probabilistic seismic hazard and risk of nuclear power plant," Advances in Civil Engineering, vol. 2018, Article ID 2698319, 18 pages, 2018.

[8] A. G. Sextos, G. D. Manolis, A. Athanasiou, and N. Ioannidis, "Seismically induced uplift effects on nuclear power plants. Part 1: containment building rocking spectra," Nuclear Engineering and Design, vol. 318, pp. 276-287, 2017.

[9] N. Nakamura, S. Akita, T. Suzuki, M. Koba, S. Nakamura, and T. Nakano, "Study of ultimate seismic response and fragility evaluation of nuclear power building using nonlinear threedimensional finite element model," Nuclear Engineering and Design, vol. 240, no. 1, pp. 166-180, 2010.

[10] J.-m. Kim, E.-h. Lee, and S.-h. Lee, "Boundary reaction method for nonlinear analysis of soil-structure interaction under earthquake loads," Soil Dynamics and Earthquake Engineering, vol. 89, pp. 85-90, 2016.

[11] Z. Wang and D. Yang, "Numerical simulation of dynamic response of nuclear island under seismic load," Rock and Soil Mechanics, vol. 34, pp. 400-406, 2013.

[12] X. Li, X. Wang, and G. Wang, "Seismic response analysis of nuclear power plant CAP1400 structure in non-rock site," Journal of Basic Science and Engineering, vol. 25, no. 32, pp. 584-594, 2017.
[13] H. Wang, J. Li, T. Tao, C. Wang, and A. Li, "Influence of apparent wave velocity on seismic performance of a superlong-span triple-tower suspension bridge," Advances in Mechanical Engineering, vol. 7, no. 6, 2015.

[14] X. Du and X. Shen, "Numerical simulation of fluid-structure interaction of LNG prestressed storage tank under seismic influence," Computers Materials and Continua, vol. 20, no. 3, pp. 225-241, 2010.

[15] C. Song, X. Li, G. Zhou, and C. Wei, "Research on FSI effect and simplified method of PCS water tank of nuclear island building under earthquake," Progress in Nuclear Energy, vol. 100, pp. 48-59, 2017.

[16] D. Wang, C. Wu, Y. Zhang, Z. Ding, and W. Chen, "Elasticplastic behavior of AP1000 nuclear island structure under mainshock-aftershock sequences," Annals of Nuclear Energy, vol. 123, pp. 1-17, 2019.

[17] W. Chen, Y. Zhang, D. Wang, and C. Wu, "Investigation on damage development of AP1000 nuclear power plant in strong ground motions with numerical simulation," Nuclear Engineering and Technology, vol. 51, no. 6, pp. 1669-1680, 2019.

[18] A. Duan, Z.-Z. Zhao, J. Chen, J.-R. Qian, and W.-L. Jin, "Nonlinear time history analysis of a pre-stressed concrete containment vessel model under Japan's March 11 earthquake," Computers and Concrete, vol. 13, no. 1, pp. 1-16, 2014.

[19] Y. Zhang, X. Li, and Q. He, "Finite element analysis of a containment vessel structures based on the multi-layer shell elements,” Building Science, vol. 30, no. 5, pp. 35-40, 2014.

[20] P. Yi, Q. Wang, and X. Kong, "Aseismic safety analysis of a prestressed concrete containment vessel for CPR1000 nuclear power plant," Earthquake Engineering and Engineering Vibration, vol. 16, no. 1, pp. 55-67, 2017.

[21] D. Wang, C. Zhuang, and Y. Zhang, "Seismic response characteristics of base-isolated AP1000 nuclear shield building subjected to beyond-design basis earthquake shaking," Nuclear Engineering and Technology, vol. 50, no. 1, pp. 170-181, 2018.

[22] X. Li, C. Song, G. Zhou, C. Wei, and M. Lu, "Experimental and numerical studies on sloshing dynamics of PCS water tank of nuclear island building," Science and Technology of Nuclear Installations, vol. 2018, Article ID 5094810, 13 pages, 2018.

[23] A. Rodriguez-Marek, E. M. Rathje, J. J. Bommer, F. Scherbaum, and P. J. Stafford, "Application of single-station sigma and site-response characterization in a probabilistic seismic-hazard analysis for a new nuclear site," Bulletin of the Seismological Society of America, vol. 104, no. 4, pp. 1601-1619, 2014.

[24] A. Lessi-Cheimariou, I. J. Tromans, E. Rathje, and C. Robertson, "Sensitivity of surface hazard to different factors and site response analysis approaches: a case study for a soft rock site," Bulletin of Earthquake Engineering, vol. 17, no. 1, pp. 73-96, 2019.

[25] R. E. Rettger, "Experiments on soft-rock deformation," AAPG Bulletin, vol. 19, no. 2, pp. 271-292, 1935.

[26] D. Zhao, B. Ruan, and G. Chen, "Validation of modified irregular loading-unloading rules based on Davidenkov skeleton curve and its equivalent shear strain algorithm implemented in ABAQUS," Chinese Journal of Geotechnical Engineering, vol. 39, no. 5, pp. 888-895, 2017.

[27] B. Ruan, K. Zhao, S. Wang, G. Chen, and H. Wang, "Numerical modeling of seismic site effects in a shallow estuarine bay (Suai Bay, Shantou, China)," Engineering Geology, vol. 260, Article ID 105233, 2019. 
[28] Y. Miao, E. Yao, B. Ruan, and H. Zhuang, "Seismic response of shield tunnel subjected to spatially varying earthquake ground motions," Tunnelling and Underground Space Technology, vol. 77, pp. 216-226, 2018.

[29] G. Chen, B. Ruan, K. Zhao et al., "Nonlinear response characteristics of undersea shield tunnel subjected to strong earthquake motions," Journal of Earthquake Engineering, vol. 24, no. 3, pp. 351-380, 2020.

[30] B. Ruan, Y. Miao, K. Cheng, and E.-1. Yao, "Study on the small strain shear modulus of saturated sand-fines mixtures by bender element test," European Journal of Environmental and Civil Engineering, vol. 2018, pp. 1-11, 2018.

[31] P. P. Martin and H. B. Seed, "One-dimensional dynamic ground response analyses," Journal of the Geotechnical Engineering Division, vol. 108, no. 7, pp. 935-952, 1982.

[32] R. L. Kuhlemeyer and J. Lysmer, "Finite element method accuracy for wave propagation problems," Journal of Soil Mechanics and Foundations Division, vol. 99, no. 5, pp. 421427, 1973.

[33] J. Liu, H. Tan, X. Bao, D. Wang, and S. Li, "Seismic wave input method for three-dimensional soil-structure dynamic interaction analysis based on the substructure of artificial boundaries," Earthquake Engineering and Engineering Vibration, vol. 18, no. 4, pp. 747-758, 2019.

[34] L. Y. Peng, Y. J. Kang, Z. Y. Tang, and H. T. Chen, "Seismic performance of CAP1400 nuclear power station considering foundation uplift," Shock and Vibration, vol. 2018, Article ID 8761209, 16 pages, 2018.

[35] H. Roh, H. Lee, and J. S. Lee, "New lumped-mass-stick model based on modal characteristics of structures: development and application to a nuclear containment building," Earthquake Engineering and Engineering Vibration, vol. 12, no. 2, pp. 307-317, 2013.

[36] H. Mogi, S. M. Shrestha, H. Kawakami, and S. Okamura, "Nonlinear soil behavior observed at vertical array in the Kashiwazaki-Kariwa nuclear power plant during the 2007 Niigata-ken Chuetsu-Oki earthquake," Bulletin of the Seismological Society of America, vol. 100, no. 2, pp. 762-775, 2010.

[37] O. V. Pavlenko and K. Irikura, "Nonlinear soil behavior at the Kashiwazaki-Kariwa nuclear power plant during the Niigata Chuetsu-Oki earthquake (July 16, 2007)," Pure and Applied Geophysics, vol. 169, no. 10, pp. 1777-1800, 2012.

[38] S. Kamagata and I. Takewaki, "New insights into seismic behavior of building and surrounding soil at Hamaoka nuclear power station during Suruga Bay earthquake in 2009," Soil Dynamics and Earthquake Engineering, vol. 53, pp. 73-91, 2013.

[39] American Society of Civil Engineers, Seismic Analysis of Safety-Related Nuclear Structures, ASCE/SEI Standard, Reston, VA, USA, 2017. 